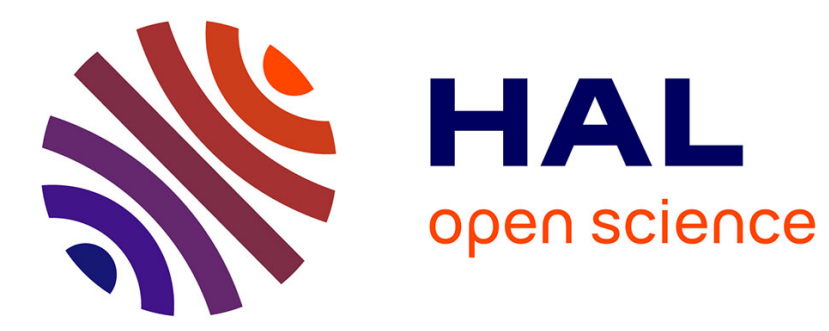

\title{
Une analyse du partage intrafamilial du revenu à partir de données subjectives
}

Ekaterina Kalugina, Natalia Radtchenko, Catherine Sofer

\section{To cite this version:}

Ekaterina Kalugina, Natalia Radtchenko, Catherine Sofer. Une analyse du partage intrafamilial du revenu à partir de données subjectives. Economie et Prévision, 2008, 186, pp.101-116. halshs00193481

\section{HAL Id: halshs-00193481 https://shs.hal.science/halshs-00193481}

Submitted on 3 Dec 2007

HAL is a multi-disciplinary open access archive for the deposit and dissemination of scientific research documents, whether they are published or not. The documents may come from teaching and research institutions in France or abroad, or from public or private research centers.
L'archive ouverte pluridisciplinaire HAL, est destinée au dépôt et à la diffusion de documents scientifiques de niveau recherche, publiés ou non, émanant des établissements d'enseignement et de recherche français ou étrangers, des laboratoires publics ou privés. 


\title{
Une analyse du partage intrafamilial du revenu à partir de données subjectives.
}

\author{
Ekaterina Kalugina* \\ Natalia Radtchenko ** \\ Catherine Sofer $^{* *}$
}

Cette étude analyse l'inégalité intrafamiliale à l'aide de données subjectives. Nous utilisons une question relative au revenu individuel de chaque membre du ménage en faisant l'hypothèse que les réponses à cette question donnent une information pertinente sur le partage effectif du revenu au sein du ménage. A partir d'un modèle collectif incluant la production domestique, et en utilisant des données russes, le Russian Longitudinal Monitoring Survey, nous montrons que, conformément aux résultats du modèle, le rapport des taux de salaires entre les deux conjoints est un facteur déterminant dans l'explication des divergences dans les réponses quant au niveau de revenu entre les deux membres du couple. Nous trouvons également un effet significatif de la différence d'âge entre les conjoints.

\footnotetext{
* Centre d'Economie de la Sorbonne, Université de Paris 1 Panthéon Sorbonne, et Haut Collège d'Economie de Moscou

E-mail : Ekaterina.Kalugina@malix.univ-paris1.fr

${ }^{* *}$ Centre d'Economie de la Sorbonne, Université de Paris 1 Panthéon Sorbonne, CNRS

E-mail : Natalia.Radtchenko@malix. univ-paris1.fr

E-mail : Catherine.Sofer@univ-parisl.fr
} 


\section{Introduction}

La présente étude a pour but de tester directement un certain nombre d'hypothèses et de résultats des modèles de décision familiale non unitaires (modèle collectif, modèle coopératif,) à l'aide de données subjectives, et de vérifier la pertinence de l'application de ce type de modèle à l'analyse des inégalités intrafamiliales. Tout d'abord, en effet, les modèles collectifs, fondés sur l'hypothèse centrale d'une pareto-optimalité des décisions et des allocations au sein du couple (Chiappori, 1988 et 1992 ; Bourguignon et Chiappori, 1992 ; Browning et Chiappori, 1998 ; Moreau et Donni, 2002) montrent qu'un pouvoir de décision inégal entre mari et femme peut se traduire par un partage inégal du revenu et des consommations dans le ménage. Il en va de même des modèles de négociations familiales fondés sur les jeux coopératifs (Manser et Brown, 1980; McElroy et Horney, 1981 ; Lundberg et Pollak, 1996).

Notons que notre papier n'a pas pour objectif de présenter un test standard du modèle collectif qui vérifierait, à partir d'un certain nombre de restrictions, que la rationalité collective n'est pas réfutée, alors que le modèle unitaire l'est : d'une part ce test a déjà été réalisé maintenant dans des dizaines d'articles, mais, d'autre part, on peut noter que ce qui est testé dans ce cas là est simultanément la rationalité collective et la spécification choisie pour l'offre de travail ou les demandes de biens. Donc le résultat de ces tests est totalement dépendant des spécifications choisies. On peut noter également que, même dans le cas où la rationalité collective fait l'objet d'un test, ce n'est très généralement pas le cas d'une autre hypothèse fondamentale du modèle, qui est celle de la Pareto-optimalité.

L'originalité de notre papier est qu'il teste directement le pouvoir de négociation de chaque conjoint, en tant que fonction d'un certain nombre de variables, sans faire d'hypothèse particulière sur la forme des offres de travail, ou des demandes. Partant de l'hypothèse (non directement testée ici, pas plus que la Pareto-optimalité, ici ou ailleurs) qu'on peut utiliser un modèle collectif pour décrire le comportement du ménage, hypothèse qui n'a pas été sérieusement réfutée, à notre connaissance, dans la littérature, nous montrons que les variables censées, dans le modèle, influencer la règle de partage, exercent effectivement un effet, dans le sens attendu, et nous examinons en outre les effets d'un certain nombre d'autres variables. Par ailleurs, les données subjectives sont de plus en plus utilisées dans les études empiriques, en particulier sur le bien-être, le bonheur ou encore la pauvreté. (Ferrer-iCarbonell et Frijters, 2004). Les données utilisées ici sont les vagues de 1994 à 1998 de l'enquête russe RLMS (Russian Longitudinal Monitoring Survey). Ici, nous évaluons les 
inégalités de revenu individuel à partir de la réponse à la question suivante : "Imaginez une échelle à neuf paliers où sur le palier le plus bas se trouvent les gens les plus pauvres et sur le palier le plus haut les gens les plus riches. Sur quel palier vous placeriez-vous?". Notons que cette approche est originale, même si un petit nombre d'articles récents (Bonke et Browing, 2003 ; Winkelmann, 2004) visent à étudier la répartition du bien-être à l'intérieur du ménage à partir de réponses à des questions différentes, mais également de type subjectif.

Le modèle collectif du ménage, de même que les modèles de jeu coopératifs, fait l'hypothèse que le pouvoir de décision de chaque membre du ménage dépend d'un certain nombre d'éléments, qui représentent les arguments de la pondération des utilités, tout d'abord, et de la règle de partage, ensuite. Les variables généralement retenues sont les salaires, les revenus individuels hors travail, et des variables appelées «facteurs de distribution » (Chiappori, Fortin et Lacroix, 2002) ou encore « variables d'environnement extra-familiales » (Mc Elroy et Horney, 1981) dont on peut penser qu'elles influencent le pouvoir de négociation de chaque membre sans exercer d'effet, ni sur les préférences, ni sur les prix relatifs. Nous testons ici l'effet des salaires et d'un certain nombre de facteurs de distribution, mais nous examinons également si des variables liées à la production non marchande sont susceptibles d'affecter le partage au sein du couple. Remarquons que si nous avons choisi de partir du cadre théorique d'un modèle collectif, nos résultats s'étendent en réalité à tout modèle faisant intervenir une négociation entre conjoints et dont l'issue dépend du pouvoir de négociation de chacun.

Nous utilisons ici un modèle collectif avec production domestique (Apps et Rees, 1997 ; Chiappori, 1997 ; Rapoport Sofer et Solaz, 2003 et 2006, Couprie, 2003), qui, d'une part, comporte une définition plus satisfaisante du loisir que les modèles d'offre de travail plus standards (Clark, Couprie et Sofer, 2004), et, d'autre part, nous permettra d'analyser les effets sur le partage au sein du ménage de variables liées à la production non marchande. Nous interprétons l'égalité intrafamiliale comme une répartition égale du revenu total, ou complet, entre les deux principaux membres du ménage. Pour cela, nous faisons l'hypothèse que les réponses à la question relative au revenu individuel fournissent une information objectivement interprétable sur le partage effectif du revenu au sein du ménage, et donc sur la règle de partage. 
Nous définissons à partir des réponses à cette question un indice d'inégalité intrafamiliale, qui est croissant avec l'augmentation relative du revenu déclaré par la femme (c.a.d. croissant avec la valeur prise par la différence entre la réponse de la femme et celle de son conjoint). Utilisant les données de l'enquête RLMS, nous estimons ensuite les valeurs prises par cet indice à partir d'un modèle de probit ordonné. L'équation de probit ordonné avec l'indice d'inégalité intrafamiliale comme variable dépendante est directement déduite de la règle de partage. Mais puisque l'allocation du temps est endogène dans le modèle, l'introduction de la production domestique nécessite l'endogénéisation du profit de la production domestique, $\Pi$. Nous ajoutons donc deux équations d'offre de travail domestique pour le mari et sa femme, qui permettent également de tester indirectement l'influence sur la règle de partage de variables de productivité non marchande. L'ensemble est estimé par la méthode du maximum de vraisemblance.

Nos résultats confirment l'influence sur le partage du revenu de variables telles que les salaires relatifs. Ils montrent également que d'autres facteurs, comme la différence d'âge entre les conjoints, que l'on peut interpréter comme des facteurs de distribution, exercent un effet sur le partage. Ils montrent enfin que, bien que l'hypothèse en soit le plus souvent faite dans les modèles, des variables plutôt liées aux aspects non marchands semblent jouer également un rôle non négligeables sur la règle de partage.

Après l'introduction (paragraphe 1), le deuxième paragraphe est consacré à la présentation du modèle collectif avec production domestique et à ses prédictions en matière de partage du revenu. Dans le troisième paragraphe, nous présentons les données et discutons la pertinence des hypothèses faites dans la suite en ce qui concerne le partage du revenu. Le quatrième paragraphe présente le modèle économétrique et le résultat des estimations. Enfin, le cinquième paragraphe conclut.

\section{Le modèle collectif avec production domestique.}

\subsection{Le modèle}

Le modèle dit collectif est fondé sur la seule hypothèse de pareto-optimalité de l'issue de la négociation. Ainsi, à l'équilibre, il n'est pas possible d'améliorer le bien-être d'un membre du 
ménage sans détériorer celui de son partenaire. L'argument principal mis en avant est que le fait même de vivre en couple suppose un désir, au moins minimal, de coopération (Clark, Couprie et Sofer, 2004). L'hypothèse d'efficacité permet de satisfaire aux exigences de testabilité, d'identifiabilité et de compatibilité avec les données.

Sur le plan théorique, l'approche générale du modèle collectif consiste à ramener le processus de décision familiale (le plus souvent avec deux conjoints) à deux étapes (Chiappori, 1988) :

1. La mise en place d'une règle de partage du revenu dépendant des préférences individuelles et du pouvoir de négociation de chacun;

2. La maximisation, de manière indépendante, de l'utilité de chaque conjoint sous la contrainte de budget découlant du partage réalisé à l'étape 1.

Partant du modèle initial, assez restrictif (Chiappori, 1988 et 1992), les travaux récents visent à élargir les hypothèses initiales en prenant en compte les questions de taxation (Donni 2003 ; Moreau et Donni, 2002), de sélection en matière d'offre de travail (Blundell et al., 1998), les biens publics (Fong et Zhang, 2001), les ménages comportant plus de deux décideurs (Fortin et Lacroix, 1997) et, enfin, la production domestique (Apps et Rees, 1997 et 1999 ; Aronsson, Daunfeldt et Wickström, 2001 ; Chiappori, 1997 ; Couprie, 2003 ; Rapoport, Sofer et Solaz, 2003 et 2006).

La production domestique a été introduite dans les modèles collectifs par Apps et Rees (1997) et Chiappori (1997). Leur modèle introduit la production domestique sous la forme d'une technologie fournissant un seul bien domestique agrégé. La modélisation de Rapoport, Sofer et Solaz (2003 et 2006) s'applique à la production d'un vecteur de biens domestiques. La présentation de notre modèle s'appuie sur celle de ces derniers articles.

Considérons un ménage composé de deux individus ( $i=$ fpour femme, $m$ pour homme). Nous étudions les couples avec ou sans enfants et avec ou sans parents âgés ${ }^{1}$, mais où seuls les deux principaux adultes du ménage disposent d'un pouvoir de négociation au sein de la famille. Chaque conjoint est doté d'une fonction d'utilité standard prenant comme arguments les consommations inobservées d'un bien composite de type Hicksien $C_{\mathrm{i}}$ avec un prix unitaire normalisé à 1 , les consommations de loisirs assignables et observées $L_{\mathrm{i}}$, et un vecteur de $\mathrm{K}$

\footnotetext{
${ }^{1}$ La présence de parents âgés dans le ménage n'est pas rare en Russie
} 
biens domestiques $\mathbf{Y}^{\mathbf{c}}$. Le ménage produit par ailleurs un vecteur de $\mathrm{K}$ biens domestiques $\mathbf{Y}^{\mathbf{p}}$. Les biens domestiques sont supposés échangeables sur le marché, c'est à dire qu'ils peuvent être vendus ou achetés en quantité illimitée aux prix unitaires établis par le marché et représentés par le vecteur p (cf. Rapoport, Sofer et Solaz, 2003 et 2006). De sorte qu'en général, $\mathbf{Y}^{\mathbf{c}} \neq \mathbf{Y}^{\mathbf{p}}$. Chaque bien domestique ${ }^{2} k$ est produit à partir d'une fonction de production à rendements décroissants qui fait intervenir comme variables le temps de travail domestique de chacun des conjoints. Nous supposerons ici que les deux membres du ménage travaillent sur le marché.

Dans un modèle collectif avec production domestique, le processus de décision Paretoefficient suppose que les membres du ménages se mettent d'accord, d'une part, sur un plan de production efficace, et, d'autre part, sur une allocation des ressources totales. Dans une première étape, le ménage détermine donc l'allocation optimale du temps de chacun de ses membres dans la production domestique, à partir de la maximisation du "profit", ou de la valeur nette de la production domestique. Ce profit est alors ajouté aux autres flux de revenu (travail marchand et revenu hors travail). Dans une seconde étape, la consommation est décentralisée une fois réalisé le choix approprié des parts $\Phi_{i}(i=f, m)$ attribuées à chacun du plein revenu.

\section{Notons :}

$t_{i}=\sum_{k} t_{i}^{k}(i=f, m)$ le temps total consacré à la production domestique par le membre $i$,

Ainsi le programme de maximisation peut être formulé de la manière suivante :

$$
\begin{aligned}
& \underset{t_{f}, t_{m}}{\operatorname{Max}} \Pi=\mathbf{p} \mathbf{Y}^{p}-w_{f} t_{f}-w_{m} t_{m} \\
& \underset{C_{i}, L_{i}, Y_{i}}{\operatorname{Max}} U_{i}\left(L_{i}, C_{i}, \mathbf{Y}^{c}{ }_{i, \ldots ;}, \mathbf{z}\right), \quad i=f, m
\end{aligned}
$$

sous contraintes budgétaire et temporaire :

$$
C_{i}+\mathbf{p} \mathbf{Y}^{c}{ }_{i}+L_{i} w_{i} \leq \Phi_{i}
$$

\footnotetext{
${ }^{2}$ Nous supposons qu'il n'existe pas de production jointe dans le secteur de la production du ménage.
} 


$$
L_{i}+h_{i}+t_{i}=T
$$

Où $w_{f}$ et $w_{m}$ sont les salaires horaires respectifs de la femme et de l'homme, $h_{i}-$ le temps de travail marchand, $\mathrm{T}$ - le temps total disponible et $\mathbf{z}$ - un vecteur représentant la part de l'hétérogénéité individuelle dans l'utilité et $\Phi_{i}\left(w_{f}, w_{m}, \mathbf{p}, y ; \mathbf{s}, \mathbf{z}\right)$ est la "règle de partage" car elle détermine les parts du plein revenu du ménage qui vont être allouées à chacun de ses membres $i$ avec :

$$
\Phi=\Phi_{f+} \Phi_{m}=\left(w_{f}+w_{m}\right) T+y+\Pi
$$

où $\mathbf{s}$ est le vecteur des facteurs de distribution ${ }^{3}, y$ est le revenu hors travail du ménage et $\Pi$ désigne le profit tiré de la production domestique. Tous les ménages étant confrontés au même vecteur de prix p, fixé sur le marché par hypothèse, des biens domestiques échangeables, celui-ci sera omis ci-dessous des fonctions endogènes du modèle.

On déduit du modèle, en particulier, les fonctions marshalliennes de demande de loisirs de la femme (3) et de l'homme (4) :

$$
\begin{aligned}
& L_{f}=L^{f}\left(w_{f}, \Phi_{f}\left(w_{f}, w_{m}, y, \mathbf{s}, \mathbf{z}\right) ; \mathbf{z}\right) \\
& L_{m}=L^{m}\left(w_{m}, y+\Pi-\Phi_{f}\left(w_{f}, w_{m}, y, \mathbf{s}, \mathbf{z}\right) ; \mathbf{z}\right)
\end{aligned}
$$

\subsection{Les comparaisons de revenu à l'intérieur du ménage.}

L'égalité au sein du ménage peut être comprise de plusieurs manières. Si nous considérons, par exemple, que des comparaisons interpersonnelles des niveaux d'utilité sont possibles, nous pouvons supposer que l'égalité à l'intérieur du ménage signifie le même niveau d'utilité des deux conjoints. Dans cette étude, nous utilisons une question subjective portant, non sur le niveau de bien-être, mais sur le revenu individuel. Dans l'enquête que nous utilisons, on demande aux individus de situer leur revenu individuel sur une échelle comportant neuf

\footnotetext{
${ }^{3}$ Les facteurs de distribution n'ont pas d'impact sur les préférences individuelles ou les prix relatifs mais influencent la répartition des pouvoirs de négociation entre les différents protagonistes (Chiappori, Fortin et Lactoix, 2002).
} 
paliers. En faisant les hypothèses habituelles (rappelées ci-dessous) permettant l'interprétation des données subjectives, nous établissons un lien direct entre les réponses à cette question et les niveaux effectifs de revenu reçus par les conjoints à l'intérieur du ménage. L'hypothèse principale est donc la suivante: les réponses individuelles à la question nous donnent l'information recherchée sur le partage effectif du plein revenu $\Phi$ au sein du ménage. Nous supposons que l'égalité intrafamiliale est définie comme le partage égal du plein revenu entre les deux conjoints. Nous considérons que le revenu est partagé de manière d'autant plus égalitaire que la femme et l'homme donnent une réponse similaire et d'autant plus inégalitaire que l'écart entre la réponse de l'un et de l'autre s'accroît. Plus précisément, nous supposons que l'écart entre la réponse donnée par la femme et celle donnée par son conjoint à la question portant sur le revenu est une fonction croissante de $\Phi_{f}-\Phi_{m}$.

\section{L'utilisation de données subjectives}

\subsection{Les données}

Les données de RLMS (Russia Longitudinal Monitoring Survey) ${ }^{4}$ sont considérées comme l'une des principales et des meilleures sources de données sur la Russie. Ce projet entrepris en 1992 a déjà fourni 13 vagues de données (1992-2004). Il s'agit d'une enquête à l'échelle nationale représentative de la situation des ménages qui est composée de deux phases distinctes : la phase I qui couvre les vagues de 1 à 4 entre 1992 et 1994 et la phase II qui couvre les vagues de 5 à 13 entre 1994 et 2004. La phase I et la phase II correspondent à deux panels de populations distinctes. Cette base de données est très riche, outre les questions de travail, notamment en ce qui concerne les indicateurs subjectifs de bien-être, les questions d'attitude sur différents sujets d'actualité en Russie (les réformes du marché, les hommes politiques, la religion...) ainsi que sur la santé. De nombreuses recherches, portant par exemple sur l'offre de travail et l'emploi en Russie sont fondées sur ces données (cf. par exemple, Earle et Sabirianova, 2002 ; Najman et Pailhé, 2001). D'autres sont plus axées sur la pauvreté (Kalugina et Najman, 2003; Ravallion et Lokshin, 2000; Senik, 2004; Zohoori et al., 1998).

\footnotetext{
${ }^{4}$ Toute l'information sur les données de RLMS peut être trouvée sur la page web du projet où on peut obtenir les données gratuitement : http://www.cpc.unc.edu/rlms.
} 
La base se compose de deux questionnaires distincts : un questionnaire ménage et un questionnaire individuel. Le premier comporte des informations relatives à la composition du ménage, les conditions de vie, la possession de biens durables, l'utilisation des terres et la production agricole du ménage à la fois consommée et vendue, les dépenses et les revenus du ménage y compris la production domestique et la réception de quelques prestations sociales; les transferts privées reçus et donnés, la région et le type de résidence.

Le questionnaire individuel interroge les individus sur leur emploi (type d'emploi, nombre d'heures travaillées, salaire, taille, structure et type de propriété de l'entreprise, information sur les arriérés et les paiements en nature), l'exploitation d'un lopin de terre (seulement jusqu'en 1998), sexe, âge, nombre d'enfants et niveau d'éducation.

Plusieurs défauts peuvent être évoqués. Dans le cadre de l'enquête RLMS, seuls sont suivis les individus qui ne changent pas d'adresse. Pourtant, la plupart des travaux fondés sur cette base de données indiquent que les données de celle-ci ont une structure de panel. Cependant, comme le remarque Heeringa (1997) ce n'est pas un panel "pur". Les ménages et les individus ayant déménagé depuis les vagues précédentes ne sont pas suivis. Un autre défaut est que la plupart des données sur les revenus et les dépenses portent sur une période d'un mois (n'oublions pas que normalement, un an se passe entre deux vagues consécutives, à l'exception des années 1997 et 1999 où l'enquête n'a pas été menée).

Pour cette étude, nous utilisons les quatre premières vagues de la deuxième phase du projet : les vagues 5 à 8 comprise entre 1994 et 1998. Plus précisément, nous disposons des données pour 1994, 1995, 1996 et 1998. Même si, au moment de l'écriture de cette étude, neuf vagues de la deuxième phase étaient déjà accessibles, nous sommes contraintes de n'utiliser que les quatre premières, car nous avons besoin des données tirées des enquêtes de budget-temps, lesquelles sont indispensables au calcul des heures de travail domestique de chaque conjoint. Or, ces questionnaires n'ont été proposés que lors des quatre premières vagues de la phase II.

Le questionnaire individuel de l'emploi du temps contient des informations concernant le temps dévolu aux différentes tâches ménagères pendant les 7 derniers jours précédant l'interview. Les données sont rapportées en heures et minutes. Les occupations sont les suivantes : le travail sur le lopin de terre, la datcha, le jardin; l'achat de nourriture; la préparation de la nourriture et le lavage de la vaisselle; le nettoyage de l'appartement; le 
lavage du linge; le repassage, la garde des enfants et des parents (par exemple, le père ou la mère âgés) etc.

Notre échantillon comprend les couples où les deux conjoints travaillent, où le chef du ménage est en âge de travailler et où deux salaires sont rapportés. Cette restriction sur les couples ayant un travail marchand n'était pourtant, en principe, pas nécessaire compte tenu de nos hypothèses théoriques : comme ici, l'estimation porte sur le travail domestique seulement, l'échantillon total aurait pu être utilisé pour la partie empirique. Néanmoins, l'estimation des salaires pour les individus qui n'offraient aucun travail marchand, s'est avérée peu satisfaisante. Nous n'avons donc gardé dans l'échantillon que les couples bi-actifs. De plus, nous n'avons conservé dans l'échantillon que les ménages dans lesquels les hommes sont âgés de 16 à 59 ans, c'est à dire non retraités. En effet, de nombreux retraités ont également un emploi en Russie, mais le cumul emploi-retraite aurait pu biaiser les estimations. La sélection donne un panel non cylindré de 1480 ménages, et 2419 observations individuelles pour la période comprise entre 1994 et 1998. Après avoir exclu pour les estimations les ménages pour lesquels les valeurs des variables nécessaires sont manquantes, l'échantillon utilisé pour l'estimation finale est composé de 1916 observations. Par ailleurs, le tableau 1 présente les fréquences du nombre de fois où un ménage est observé dans le panel. Il en résulte que plus de la moitié des ménages est présente seulement une fois dans le panel, tandis que seulement $16,8 \%$ de l'échantillon y est observé au moins trois fois. Cette qualité médiocre de l'enquête en tant que panel, peut s'expliquer non seulement par le taux d'attrition élevé dans le cadre des données RLMS, mais aussi par la force de nos exigences : nous ne retenons que les couples pour lesquels deux salaires sont observables, ce qui est une grande restriction, surtout dans le cas de la Russie où les individus préfèrent souvent ne pas déclarer leurs salaires. Ces résultats nous ont conduites à utiliser les données empilées pour nos estimations. A ce stade de notre travail, nous n'utilisons pas, en effet, (sauf cas particulier, cf ci-dessous), la double dimension des données de panel (temporelle et individuelle), c'est-à-dire que nous ne prenons pas en compte les spécificités inobservables, stables dans le temps. En revanche, nous introduisons les variables-indicatrices de l'année de l'observation qui sont supposées capter les changements macroéconomiques en Russie pendant la période de transition.

Tableau 1. Fréquence du nombre d'observations par ménage. 


\begin{tabular}{cc}
\hline 1 & 55.6 \\
2 & 27.6 \\
3 & 12.4 \\
4 & 4.4 \\
\hline Total & 100 \\
\hline
\end{tabular}

Source : RLMS (vagues 5-8)

Le tableau 2 donne les moyennes ainsi que les écarts-types de quelques variables clés utilisées dans l'analyse économétrique, présentées séparément pour les hommes et pour les femmes. Les taux de salaires pour chaque période sont exprimés en roubles.

Tableau 2. Statistiques descriptives de l'échantillon

\begin{tabular}{|c|c|c|c|c|c|c|}
\hline \multirow[b]{2}{*}{ Variable } & \multicolumn{3}{|c|}{ Femmes } & \multicolumn{3}{|c|}{ Hommes } \\
\hline & $\begin{array}{c}1995 \\
\text { Moyenne } \\
\text { (écart-types) }\end{array}$ & $\begin{array}{c}1996 \\
\text { Moyenne } \\
\text { (écart-types) }\end{array}$ & $\begin{array}{c}1998 \\
\text { Moyenne } \\
\text { (écart-types) }\end{array}$ & $\begin{array}{c}1995 \\
\text { Moyenne } \\
\text { (écart-types) }\end{array}$ & $\begin{array}{c}1996 \\
\text { Moyenne } \\
\text { (écart-types) }\end{array}$ & $\begin{array}{c}1998 \\
\text { Moyenne } \\
\text { (écart-types) }\end{array}$ \\
\hline $\begin{array}{l}\text { Travail marchand par } \\
\text { semaine }\left(h_{\mathrm{i}}\right), \text { hrs }\end{array}$ & $\begin{array}{c}\mathbf{3 9 . 5} \\
(12.23)\end{array}$ & $\begin{array}{c}\mathbf{3 8 . 4 1} \\
(14.68)\end{array}$ & $\begin{array}{c}\mathbf{3 8 . 7 8} \\
(15.38)\end{array}$ & $\begin{array}{l}\mathbf{4 5 . 2 2} \\
(12.99)\end{array}$ & $\begin{array}{c}\mathbf{4 4 . 7 5} \\
(16.84)\end{array}$ & $\begin{array}{l}\mathbf{4 4 . 7 2} \\
(17.25)\end{array}$ \\
\hline $\begin{array}{l}\text { Travail domestique par } \\
\text { semaine }\left(h h_{\text {j }}\right) \text { hrs }\end{array}$ & $\begin{array}{l}42.9 \\
(30.4)\end{array}$ & $\begin{array}{c}45 \\
(30.7)\end{array}$ & $\begin{array}{l}\mathbf{4 6 . 8 7} \\
(29.8)\end{array}$ & $\begin{array}{c}\mathbf{1 3 . 7 4} \\
(17.52)\end{array}$ & $\begin{array}{c}\mathbf{1 5 . 7 1} \\
(19.36)\end{array}$ & $\begin{array}{c}14.72 \\
(16.47)\end{array}$ \\
\hline $\begin{array}{l}\text { Travail total par semaine }\left(H_{\mathrm{i}}\right) \text {, } \\
\text { hrs }\end{array}$ & $\begin{array}{c}\mathbf{8 2 . 3 6} \\
(31.53)\end{array}$ & $\begin{array}{c}\mathbf{8 3 . 2 3} \\
(31.49)\end{array}$ & $\begin{array}{c}\mathbf{8 5 . 6 6} \\
(31.92)\end{array}$ & $\begin{array}{c}\mathbf{5 8 . 9 5} \\
(22.48)\end{array}$ & $\begin{array}{c}\mathbf{6 0 . 4 3} \\
(24.93)\end{array}$ & $\begin{array}{l}\mathbf{5 9 . 3 9} \\
(22.69)\end{array}$ \\
\hline $\begin{array}{l}\text { Taux de salaire horaire }\left(w_{\mathrm{i}}\right) \text {, } \\
\text { roubles }\end{array}$ & $\begin{array}{c}7 \\
(17)\end{array}$ & $\begin{array}{c}\mathbf{6 . 1 2} \\
(14,5)\end{array}$ & $\begin{array}{c}3.87 \\
(10.9)\end{array}$ & $\begin{array}{c}\mathbf{1 2 . 7} \\
(55.5)\end{array}$ & $\begin{array}{c}\mathbf{1 0 . 4 6} \\
(36)\end{array}$ & $\begin{array}{c}7.8 \\
(45)\end{array}$ \\
\hline $\begin{array}{l}\text { Revenu total mensuel }(Y) \text {, } \\
\text { roubles }\end{array}$ & $\begin{array}{l}\mathbf{2 8 8 7} \\
(6145)\end{array}$ & $\begin{array}{l}2696 \\
(5878)\end{array}$ & $\begin{array}{c}2196 \\
(14484)\end{array}$ & $\begin{array}{l}\mathbf{2 8 8 7} \\
(6145)\end{array}$ & $\begin{array}{l}\mathbf{2 6 9 6} \\
(5878)\end{array}$ & $\begin{array}{c}2196 \\
(14484)\end{array}$ \\
\hline
\end{tabular}

Source : RLMS (vagues 6-8).

Nous remarquons en premier lieu une énorme différence entre le nombre d'heures de travail domestique entre les hommes et les femmes. En effet, bien que les femmes travaillent un peu moins longtemps sur le marché (la différence est approximativement de 6 heures par semaines), le nombre d'heures consacrées au travail domestique par les femmes est trois fois supérieur à celui des hommes. Nous ne pouvons comparer nos résultats qu'à la recherche de Mezentseva (2000) sur le travail domestique en Russie. Elle utilise la même base de données et rapporte des indicateurs pour l'année 1998. Selon ses calculs, bien que le temps consacré au travail marchand ne varie pas considérablement entre les femmes et les hommes, celles-ci travaillent à la maison deux fois plus que les hommes. Elle souligne également la grande disparité du temps de travail domestique en fonction des tâches accomplies. Notons que la différence entre le nombre d'heures totales travaillées par les femmes et par les hommes est donc d'environ 25 heures par semaine. C'est considérable, et d'autant plus considérable si on compare avec la plupart des pays d'Europe dans lesquels cette différence est en général beaucoup plus faible, voire nulle (cf pour la France Rapoport, Sofer et Solaz, 2006 ou, pour le 
Danemark, Bonke et Browning, 2003, par exemple). Cet écart pourrait s'expliquer en partie, mais sans doute en faible partie seulement, par le fait qu'aucune distinction n'est faite dans le questionnaire entre activité principale et activité secondaire, ce qui conduit à les ajouter au lieu de ne garder, comme c'est d'usage, que les activités déclarées comme principales.

\subsection{Le revenu subjectif auto déclaré et son interprétation.}

L'utilisation des données subjectives, initiée principalement par l'école de Leyde pour la mesure subjective de la pauvreté dans les années 1970, ne cesse de se développer. De nombreuses enquêtes nationales comme British Household Panel Survey (BHPS), le German Socio-Economic Panel (GSOEP) ou les données russes utilisées ici comprennent désormais des questions subjectives concernant la satisfaction de la vie en général, de l'emploi, de la santé, de la situation financière, de l'inégalité etc. Ces réponses sont le plus souvent qualitatives et prennent des valeurs discrètes, par exemple comprises entre 0 et 10 . Elles sont généralement utilisées comme proxy du bien-être individuel (Senik, 2005) et sont estimées par des modèles économétriques afin de trouver les relations entre le bien-être individuel subjectif et des variables objectives, comme le revenu, le statut sur le marché du travail, etc.

Pourquoi utiliser des données subjectives? Par exemple, Senik (2002) l'explique de la manière suivante : "Le recours aux données subjectives se justifie essentiellement par les limites de la démarche positive fondée sur la révélation des préférences. Il ne s'agit pas de remettre en cause le caractère central de cette dernière au sein de la théorie économique, mais de la compléter dans les cas où son champ d'application est limité, en particulier lorsque les défaillances du marché (externalités), les interactions hors marché et les défauts de coordination des actions individuelles interdisent de retracer le lien entre préférences et résultats de l'action individuelle." Ainsi, le recours aux données subjectives se justifie par l'absence d'autres moyens pour analyser les choix individuels et éclairer les politiques publiques. Par exemple, des informations subjectives ont été utilisées afin d'analyser les coûts non monétaires du chômage (Clark et Oswald, 1994 ; Winkelmann et Winkelmann, 1998) ou afin de découvrir les attitudes individuelles vis-à-vis de l'inégalité (Ng; 1996 ; Ravallion et Lokshin, 2000). En général, ce type d'analyse fournit des résultats empiriques plausibles et explicables. 
Pour l'analyse des données subjectives, deux hypothèses principales doivent être faites : les individus sont capables d'évaluer leur propre situation, d'une part, et les réponses individuelles sont comparables entre elles, d'autre part (Ferrer-i-Carbonell, 2002). En d'autres termes, les individus comprennent et répondent aux questions subjectives de manière similaire. Il s'agit là d'une hypothèse forte car les individus sont différents, et ils font faces à des environnements sociaux hétérogènes, etc. Pourtant, les résultats empiriques indiquent qu'au sein d'une communauté parlant la même langue, les individus ont une compréhension similaire des concepts tels que le bien-être, le bonheur, etc. (Van Praag, 1991). Dans notre cas, il est raisonnable de supposer que les membres d'un même ménage ont le même environnement social et partagent donc les mêmes références concernant leurs revenus.

Afin de mesurer le revenu subjectif, nous utilisons la question suivante : "Imaginez une échelle à neuf paliers où sur le palier le plus bas se trouvent les gens les plus pauvres et sur le palier le plus haut les gens les plus riches. Sur quel palier vous placeriez-vous?". Cette question fait partie du questionnaire individuel (par opposition au questionnaire ménage) et est posée aux différents membres du ménage. Nous avons déjà souligné qu'à partir des données subjectives, le chercheur peut acquérir une information indisponible autrement. L'inégalité intrafamiliale nous semble être un domaine pour lequel ce critère est approprié.

Le tableau 3 présente les statistiques descriptives relatives à l'échantillon des individus qui ont répondu à la question subjective et pour qui les salaires sont non nuls et non manquants. Nous avons regroupés les paliers 6, 7,8 et 9 car le nombre d'observations dans ces catégories est très réduit. Le tableau 3 montre la répartition des réponses à la question subjective. Un pourcentage non négligeable de la population se déclare pauvre. Si nous admettons que les pauvres sont ceux se situant sur les deux premiers paliers de l'échelle, le taux de pauvreté subjective passe de 19,53\% en 1994 à $24,31 \%$ en 1998. La plupart des individus se concentrent pourtant sur les paliers 3,4 et 5 .

Tableau 3. Les revenus auto déclarés.

\begin{tabular}{lccccc}
\hline $\begin{array}{l}\text { Question subjective } \\
\text { 1- les plus pauvres; 6 et } \\
\text { plus - les plus riches }\end{array}$ & Vague 5(1994) & Vague 6(1995) & Vague 7(1996) & Vague 8(1998) & $\begin{array}{c}\text { Total } \\
\text { échantillon } \\
\text { Nombre }(\%)\end{array}$ \\
\hline 1 & $109(6,15)$ & $137(8,95)$ & $69(6,32)$ & $100(8,41)$ & $415(7,43)$ \\
2 & $237(13,38)$ & $181(11,82)$ & $145(13,28)$ & $189(15,90)$ & $752(13,47)$ \\
3 & $467(26,37)$ & $338(22,08)$ & $260(23,81)$ & $319(26,83)$ & $1384(24,79)$ \\
4 & $442(24,96)$ & $393(25,67)$ & $273(25,00)$ & $296(24,89)$ & $1404(25,15)$ \\
\hline
\end{tabular}




\begin{tabular}{lccccc}
\hline 5 & $388(21,91)$ & $365(23,84)$ & $260(23,81)$ & $225(18,92)$ & $1238(22,17)$ \\
6 et plus & $128(7,23)$ & $117(7,64)$ & $85(7,78)$ & $60(5,05)$ & $390(6,99)$ \\
\hline Total (individus) & $1771(100)$ & $1531(100)$ & $1092(100)$ & $1189(100)$ & $5583(100)$ \\
\hline
\end{tabular}

Source : RLMS (vagues 5-8).

Echantillon : individus répondant à la question subjective

Dans cette étude, nous nous intéressons principalement aux différences de revenus à l'intérieur d'un ménage donné, en partant de l'hypothèse que les différences des réponses subjectives entre le mari et la femme sont susceptibles de refléter un partage effectivement inégal entre les deux conjoints. Le tableau 4 présente les différences dans les réponses à la question subjective pour l'homme et la femme au sein d'un même ménage. L'échantillon concerné est celui des couples où les deux conjoints ont répondu à la question subjective, et pour lesquels nous observons deux salaires.

Les écarts de plus de deux paliers étant rares, ils ont été regroupés avec les écarts correspondants à exactement deux paliers. Les différences vont donc de - 2 (la femme se déclare plus pauvre que l'homme avec au moins deux paliers d'écart) à +2 (la femme se déclare plus riche que l'homme avec au moins deux paliers d'écart).

En moyenne, dans plus de 50\% des ménages, les femmes et les hommes répondent différemment à cette question. Dans presque 20\% des ménages, les hommes se situent sur un palier plus bas que leur femme, et pour $10 \%$ des ménages, cette différence est de 2 paliers ou plus. Toutefois, les femmes ont globalement des revenus subjectifs plus faibles que ceux de leur mari : par exemple, en 1998, dans plus de 34\% des ménages, les femmes donnent des valeurs de revenus plus basses que celles de leur conjoint, contre $28 \%$ où ce sont les hommes qui se déclarent plus pauvres.

Tableau 4. Différences dans les réponses à la question subjective.

\begin{tabular}{|c|c|c|c|c|c|}
\hline $\begin{array}{l}\text { Réponse de la } \\
\text { femme - celle de } \\
\text { l'homme }\end{array}$ & $\begin{array}{c}\text { Vague5(1994) } \\
\text { Nombre }(\%)\end{array}$ & $\begin{array}{c}\text { Vague } 6(1995) \\
\text { Nombre }(\%)\end{array}$ & $\begin{array}{c}\text { Vague } 7(1996) \\
\text { Nombre }(\%)\end{array}$ & $\begin{array}{c}\text { Vague } 8(1998) \\
\text { Nombre }(\%)\end{array}$ & $\begin{array}{c}\text { Total } \\
\text { échantillon } \\
\text { Nombre }(\%)\end{array}$ \\
\hline-2 & $87(10,82)$ & $61(9,38)$ & $53(11,35)$ & $60(11,83)$ & $261(10,75)$ \\
\hline-1 & $139(17,29)$ & $126(19,38)$ & $101(21,63)$ & $112(22,09)$ & $478(19,69)$ \\
\hline 0 & $339(42,16)$ & $283(43,54)$ & $188(40,26)$ & $192(37,87)$ & $1002(41,27)$ \\
\hline 1 & $142(17,66)$ & $127(19,54)$ & $80(17,13)$ & $95(18,74)$ & $444(18,29)$ \\
\hline 2 & $97(12,06)$ & $53(8,15)$ & $45(9,64)$ & $48(9,47)$ & $243(10,01)$ \\
\hline Total des ménages & $804(100)$ & $650(100)$ & $467(100)$ & $507(100)$ & $2428(100)$ \\
\hline
\end{tabular}


Sur la base des résultats du tableau 3 , nous construisons un indice d'inégalité intrafamiliale $\mathrm{I}_{1}$ qui prend les valeurs comprises entre -2 et +2 rapportées au tableau 4 . Cependant, nous sommes conscientes qu'interpréter une petite différence entre les réponses des conjoints comme une vraie inégalité intrafamiliale est une hypothèse un peu forte. C'est pourquoi en construisant l'indice d'inégalité intrafamiliale pour les estimations empiriques, nous avons tenté diverses spécifications. Nous avons, par exemple construit un deuxième indice $I_{2}$ qui prend seulement trois valeurs $(0,1$ et 2$)$ en regroupant les réponses différentes -2 et $-1\left(I_{2}=0\right.$ dans ce cas), en gardant le groupe qui donne des réponses égales $\left(\mathrm{I}_{2}=1\right.$ dans ce cas), et en regroupant les valeurs +1 et +2 , d'autre part $\left(\mathrm{I}_{2}=2\right.$ dans ce cas). Nous avons également construit un troisième indice, $I_{3}$, qui élargit le critère d'égalité à une différence d'un seul palier entre les réponses des deux conjoints : $I_{3}$ prend également seulement trois valeurs $0,1,2$ avec une valeur de 1 si les valeurs données par les deux conjoints sont les mêmes ou diffèrent au plus d'un palier, 0 si la femme déclare un revenu plus faible que son conjoint avec au moins deux paliers d'écart, et une valeur de 2 si elle déclare un revenu plus élevé avec au moins deux paliers d'écart.

Comme signalé ci-dessus, nous considèrons dans cet article que le plein revenu du ménage est partagé de manière d'autant plus égalitaire que la femme et l'homme donnent une réponse similaire et d'autant plus inégalitaire que l'écart entre la réponse de l'un et de l'autre s'accroît. Il est à noter cependant que la question subjective utilisée est relativement vague sur la nature du revenu concerné, et la question se pose de savoir si les réponses individuelles reflètent bien une estimation de la part individuelle du plein revenu, ou bien autre chose, comme, par exemple, les revenus individuels du travail. La littérature sur les données subjectives remarque que la place dans le questionnaire de la question analysée joue un rôle dans les réponses individuelles. Il est vrai que la question qui nous intéresse est posée dans la partie «individuelle » du questionnaire, celle qui concerne les aspects individuels tels que le travail et le revenu du travail. Mais d'autre part, la question vient après une autre interrogation relative au niveau de vie de ménage anticipé pour l'année prochaine.

Pour éclairer l'interprétation de la question sur le revenu subjectif dont les réponses nous servent à construire l'indice de l'inégalité, nous présentons une estimation simultanée des réponses des deux membres de ménage, régressées sur les revenus du travail de chacun d'eux, en contrôlant pour certaines caractéristiques individuelles et de ménage. Si la réponse 
des individus était purement individuelle, et donc relative à leurs propres revenus du travail marchand, on devrait trouver une influence faible, voire nulle du revenu d'un individu sur la réponse de son partenaire.

Le résultat obtenu par la méthode 3SLS est présenté au tableau 5.

Tableau 5. Estimation simultanée des réponses de la femme et de l'homme.

\begin{tabular}{lcc}
\hline & $\begin{array}{c}\text { Réponses de la femme } \\
\text { Coefficient }\end{array}$ & $\begin{array}{c}\text { Réponses de l'homme } \\
\text { Coefficient }\end{array}$ \\
\hline Revenu (mensuel) du travail de la femme & $\mathbf{1 1 . 4 9 6 * * *}^{* *}$ & $\mathbf{3 . 6 3 7}^{* *}$ \\
Revenu (mensuel) du travail de l'homme & $\mathbf{5 . 0 4 0}^{* *}$ & $\mathbf{7 . 7 6 2}^{* * *}$ \\
Différence d'âge: Age - Age $\mathrm{m}$ & 0.000 & $0.020^{* * *}$ \\
Revenu hors travail du ménage & $0.001^{* *}$ & $0.001^{* * *}$ \\
Nombre d'enfants entre 0 et 7 ans & 0.061 & 0.074 \\
Nombre d'enfants entre 7 et 18 ans & -0.030 & -0.042 \\
Nombre de personnes âgées & $0.159^{* * *}$ & 0.036 \\
Ln de la taille du logement (en mètres carrés) & -0.009 & 0.032 \\
Ménage travaille sur un lopin de terre & -0.078 & -0.069 \\
Femme ayant un niveau d'études supérieur que & 0.031 & $-0.136^{* *}$ \\
celui de son mari & $-0.255^{* * *}$ & $-0.240^{* * *}$ \\
Moscou/Saint-Pétersbourg & 0.073 & -0.076 \\
Vague5 & 0.085 & 0.033 \\
Vague6 & -0.018 & -0.061 \\
Vague8 & $0.025^{* *}$ & $0.027^{* *}$ \\
Nombre d'années d'éducation de l'homme & $2.897^{* * *}$ & $3.059^{* * *}$ \\
Constante & 2163 & 2163 \\
\hline Nombre d'observations & 0.0703 & 0.0709 \\
$\mathrm{R}^{2}$ & 163.67 & 164.99 \\
$\chi^{2}$ & 0.0000 & 0.0000 \\
Prob. $>\chi^{2}$ & & \\
\hline
\end{tabular}

Les résultats de ce tableau indiquent bien que les membres du ménage se réfèrent bien dans leur réponse à un revenu faisant intervenir l'ensemble des revenus du ménage plutôt qu'à leur propre salaire seul. En effet, pour les deux sexes les réponses sont fortement influencées, non pas uniquement par leur propre revenu mais aussi par le revenu de leur partenaire. De plus, pour chacun, l'impact de ses propres revenus du travail est plus élevé que celui qui correspond à l'évaluation, par chacun, de la contribution de l'autre conjoint(e). Cela signifie que le poids donné, dans son revenu total, par chaque membre du ménage à ses propres revenus du travail est plus élevé que le poids évalué par le conjoint de ces mêmes revenus. Ce résultat est une première indication du caractère plausible de l'interprétation des réponses en termes de "pouvoir de négociation". 
Une autre objection à notre hypothèse que la différence d'évaluation de leur revenu par les deux membres du ménage reflète leur pouvoir de négociation fait intervenir une différence plus subjective entre les « attentes» ou les « besoins » des deux partenaires ${ }^{5}$. Des écarts dans la réponse sur les revenus reflèteraient plutôt, selon cette interprétation, des écarts entre les conjoints sur ces aspects plus psychologiques du bien-être. Bien qu'il ne soit évidemment pas possible d'écarter totalement cette possibilité, nous avons réalisé un autre test qui permet de répondre à cette objection. En effet, une autre question subjective de la base RLMS donne une indication sur le niveau de bien-être des individus. Cette question est la suivante: "A l'heure actuelle, quel est votre degré de satisfaction quant à votre vie en général?". Les réponses possibles sont les suivantes: pleinement satisfait, plutôt satisfait, moyennement satisfait, pas assez satisfait, pas satisfait du tout. Si les écarts de bien-être correspondant à des aspects psychologiques en termes d' «attente », ou de « besoin » gouvernaient principalement, ou de façon importante, la réponse sur les revenus, on peut penser que l'on devrait observer une corrélation entre les écarts relevés dans les réponses à ces deux questions

Dans le tableau 6 ci-dessous nous présentons les statistiques descriptives relatives aux différences dans les réponses concernant la satisfaction générale pour l'homme et la femme au sein d'un même ménage. On voit que, comme dans le cas de la question subjective portant sur le revenu, dans plus de 50\% des ménages, les femmes et les hommes répondent différemment à cette question.

Tableau 6. Différences dans les réponses à la question sur la satisfaction quant à la vie en général.

\begin{tabular}{lcccc}
\hline $\begin{array}{l}\text { La réponse de la femme - celle de } \\
\text { l'homme }\end{array}$ & $\begin{array}{c}\text { Vague5(1994) } \\
\text { Nombre(\%) }\end{array}$ & $\begin{array}{c}\text { Vague6(1995) } \\
\text { Nombre }(\%)\end{array}$ & $\begin{array}{c}\text { Vague7(1996) } \\
\text { Nombre }(\%)\end{array}$ & $\begin{array}{c}\text { Vague8(1998) } \\
\text { Nombre (\%) }\end{array}$ \\
\hline-2 & $80(11,07)$ & $50(8,25)$ & $39(9,11)$ & $49(10,40)$ \\
-1 & $133(18,40)$ & $139(22,94)$ & $101(23,60)$ & $120(25,48)$ \\
0 & $326(45,09)$ & $274(45,21)$ & $201(46,96)$ & $203(43,10)$ \\
1 & $137(18,95)$ & $105(17,33)$ & $61(14,25)$ & $76(16,14)$ \\
2 & $47(6,50)$ & $38(6,27)$ & $26(6,07)$ & $23(4,88)$ \\
\hline Total des ménages & $723(100)$ & $606(100)$ & $428(100)$ & $471(100)$ \\
\hline
\end{tabular}

Source : RLMS (vagues 5-8).

Echantillon : couples (les réponses de la femme et de son conjoint)

\footnotetext{
${ }^{5}$ Nous tenons à remercier un rapporteur anonyme pour cette suggestion.
} 
0 - il n'y pas de différence entre la réponse de la femme et celle de son mari, -1 - la femme si situe sur le palier inférieur à celui où se trouve son mari, $-2-1$ a femme se situe deux paliers plus bas ou plus (moins satisfaite) de celui où se trouve son mari, 1 - la femme se situe sur le palier supérieur (plus satisfaite) à celui où se trouve son mari, 2 - la femme se situe deux paliers plus haut ou plus (plus satisfaites) de celui où se trouve son mari.

Les niveaux de satisfaction indiqués peuvent être interprétés directement comme des niveaux de bien-être, ou encore d'utilité. Comme la fonction d'utilité (indirecte) est croissante par rapport au revenu, il est tout à fait naturel de supposer l'existence d'une corrélation positive entre les réponses aux deux questions. C'est bien ce que nous trouvons, pour l'ensemble des individus, avec une corrélation significative égale à 0.4 .

Le test consiste à confronter les divergences entre les évaluations de la femme et celles de l'homme obtenues par les deux questions. Les corrélations sont rapportées au tableau 7.

\section{Tableau 7. Corrélation}

\begin{tabular}{|l|c|}
\hline \multicolumn{1}{|c|}{ Variables } & Corrélation \\
\hline Réponses aux questions sur le revenu et satisfaction & $0.4^{* * *}$ \\
\hline $\begin{array}{l}\text { Divergences (entre deux partenaires) des réponses aux questions sur } \\
\text { le revenu et satisfaction }\end{array}$ & $0.17^{* * *}$ \\
\hline
\end{tabular}

$* * *$ : significatif à $1 \%$

Comme attendu, il y a une forte corrélation entre les réponses de deux questions. Par contre la corrélation entre les écarts des réponses est faible. Notamment dans le tableau 8 on peut voir les fréquences des indices IR et IS d'inégalité construite à la base des questions sur le revenu et la satisfaction respectivement.

Tableau 8.

\begin{tabular}{|c|cccc|c|}
\hline & \multicolumn{4}{|c|}{ Divergences de satisfaction (IS) } & Total \\
\hline Divergences des revenus (IR) & 0 & 1 & 2 & 250 \\
& 0 & 103 & 101 & 46 & $\mathbf{1 , 7 4 8}$ \\
$\mathbf{1}$ & $\mathbf{5 5 2}$ & $\mathbf{8 1 8}$ & $\mathbf{3 7 8}$ & 230 \\
\hline 2 & 56 & 85 & 89 & 2,228 \\
\hline Total & 711 & 1,004 & 513 & \\
\hline
\end{tabular}

D'après le tableau des fréquences, la moitié des couples donnant la même réponse à la question sur le revenu diverge en répondant à la question sur la satisfaction. Ces observations montrent que deux questions étant liées, ne sont pas équivalentes. Du point de vue de notre interprétation l'égalité des revenus n'entraîne pas effectivement l'égalité des utilités. Nous 
supposons alors que les écarts de réponses à la question sur le revenu ne concernent pas généralement les attentes ou besoins différents des membres des ménages.

Nous allons maintenant approfondir la vérification empirique de cette interprétation.

\section{Un test du pouvoir de négociation}

En supposant donc à partir de maintenant que les données subjectives apportent une information fiable en ce qui concerne les parts individuelles dans le revenu total, à savoir $\Phi_{f}$ et $\Phi_{m}$, nous pouvons tester la pertinence des effets attendus d'un certain nombre de variables sur la règle de partage.

\subsection{Le modèle économétrique}

Il s'agit donc de vérifier les effets des variables habituellement mises en avant dans les modèles sur la part du revenu allouée à chaque conjoint. Tout d'abord, nous avons effectué deux tests préalables.

Dans un premier temps, compte tenu de la formulation numérique de l'échelle des revenus, nous approximons la différence des réponses de la femme et de l'homme par une variable continue. Ainsi, nous pouvons appliquer le modèle MCO à effets fixes pour l'estimation de cette variable afin de prendre en compte les effets individuels non observés stables dans le temps. Les résultats sont rapportés au tableau 9.

Tableau 9. Estimation de l'écart entre les réponses de la femme et de l'homme par un modèle MCO à effets fixes.

\begin{tabular}{|l|c|}
\hline & Coefficient \\
\hline Ln du rapport des salaires $\ln \left(w_{f} / w_{m}\right)$ & $0.127^{*}$ \\
Ln du taux de salaire de l'homme & 0.106 \\
Différence d'âge: Age Age $_{\mathrm{m}}$ & $0.218^{*}$ \\
Revenu hors travail du ménage & 0.000 \\
Nombre d'enfants entre 0 et 7 ans & $0-.066$ \\
Nombre d'enfants entre 8 et 18 ans & -0.047 \\
Nombre de personnes âgées & $-0.344^{*}$ \\
Ln de la taille du logement (en mètres carrés) & -0.056
\end{tabular}




\begin{tabular}{|l|c|} 
Ménage travaille sur un lopin de terre & -0.109 \\
Femme ayant un niveau d'études supérieur à celui & 0.195 \\
de son mari & \\
Moscou/Saint-Pétersbourg & 0.000 \\
Vague5 & 0.158 \\
Vague6 & 0.087 \\
Vague8 & 0.082 \\
Nombre d'années d'éducation de l'homme & 0.048 \\
Constante & -0.258 \\
\hline Nombre d'observations & 2177 \\
Nombre de groupes & 1407 \\
Nombre moyen d'observations par groupe & 1.5 \\
R-sq between & 0.0016 \\
F test de ui=0: F(1406, 756)=1.22, Prob>F=0.0011 & \\
\hline *significatif à 10\%; ** significatif à 5\%; *** significatif à 1\% \\
Source : RLMS (vagues 5-8)
\end{tabular}

Le $\mathrm{R}^{2}$ «between » montre la contribution très faible des effets invariants. Cela semble bien confirmer, comme l'a déjà montré notre test relatif à la question portant sur la satisfaction, que l'hétérogénéité inobservée, dont on pourrait penser qu'elle joue un rôle important sur des réponses subjectives (effets de caractéristiques psychologiques, comme le pessimisme ou l'optimisme, par exemple) a en fait un rôle peu important.

Ensuite nous avons estimé un modèle simple de probit ordonné dans lequel la variable expliquée, qui correspond au différentiel de revenu total alloué par le ménage à la femme et à l'homme, $\Phi_{f}-\Phi_{m}$, est mesuré par l'indice $\mathrm{I}_{1}$ défini ci-dessus ${ }^{6}$. Celui-ci prend comme valeurs les cinq valeurs définies par la différence entre les réponses de la femme et celle de l'homme.

Mais le modèle économétrique correspondant au modèle théorique élaboré ci-dessus peut être formulé de façon plus adéquate comme un modèle de probit ordonné avec travail domestique endogène: en effet, l'équation de probit ordonné avec l'indice d'inégalité intrafamiliale comme variable dépendante est directement déduite de la règle de partage. Cependant, puisque l'allocation du temps est endogène dans le modèle, et que le revenu total dépend du profit de la production domestique, $\Pi$, il en va de même de la différence des revenus alloués à

\footnotetext{
${ }^{6}$ Toutes les estimations sont effectuées en prenant en compte la dépendance des observations à l'intérieur des groupes (les observations relatives aux diverses périodes pour le même individu). En spécifiant l'option « cluster» sous Stata, les observations sont supposées être indépendantes entre les goupes, mais pas nécessairement indépendantes à l'intérieur d'un groupe. Cette spécification affecte les écarts-types estimés et la matrice variance-covariance des estimateurs (VCE), mais pas les coefficients estimés.
} 
chaque conjoint, $\Phi_{f}-\Phi_{m}$. Donc, la prise en compte de la production domestique nécessite l'endogénéisation du profit qui en découle. Nous ajoutons donc deux équations d'offre de travail domestique pour le mari et sa femme. Cette deuxième partie du modèle fournit également un test de l'influence, directe ou indirecte, des caractéristiques non marchandes sur la règle de partage. Si la règle de partage est supposée être une fonction des caractéristiques monétaires (salaires, revenus hors travail, etc.), mais pas directement des variables non monétaires (comme la productivité du travail domestique par exemple) ${ }^{7}$, le modèle théorique implique que la seule corrélation possible entre l'équation de probit ordonné et chacune des deux équations d'offre de travail domestique apparaît via le profit de la production domestique. Néanmoins, la mise en évidence d'une telle corrélation pourrait aussi témoigner d'un effet plus direct des caractéristiques non marchandes sur la règle de partage.

Ce troisième modèle est estimé en utilisant comme variable dépendante l'indice $I_{3}$ défini cidessus. Rappelons que $I_{3}$ traite comme semblables les réponses différant au plus d'un palier. Les mêmes estimations, réalisées avec l'indice $I_{2}$ comme variable expliquée, dont les trois valeurs correspondent à : $0=$ inégalité stricte des réponses en défaveur de la femme, $1=$ égalité des réponses, 2 = inégalité stricte des réponses en faveur de la femme, donnent des résultats très similaires : les résultats sont donc tout à fait robustes aux différentes définitions de la différence $\Phi_{f}-\Phi_{m}$. L'estimation du modèle 3 est réalisée à partir de la méthode de Maximum de Vraisemblance avec Information Complète (cf. encadré).

\section{Encadré: Le modèle économétrique complet}

Appelons $\mathrm{I}_{3}$ la fonction-indicatrice prenant les valeurs 0,1 ou 2 selon les différences observées dans les valeurs du revenu subjectif entre les hommes et les femmes. Rappelons que, suivant la définition donnée ci-dessus de cette fonction, on a :

$$
I_{3}=\left\{\begin{array}{cc}
0, & \text { si } \Phi_{f}<\Phi_{m} \\
1, & \text { si } \Phi_{f}=\Phi_{m} \\
2, & \text { si } \Phi_{f}>\Phi_{m}
\end{array}\right.
$$

\footnotetext{
${ }^{7}$ C'est l'hypothèse faite le plus souvent (cf. Apps et Rees, 1997 ; Chiappori, 1997 ; Rappoport, Sofer et Solaz,
} 2003). 
Notons $\Phi_{f}^{*}$ la fonction correspondant à la règle de partage non observée :

$$
\Phi_{f}^{*}=\gamma \mathbf{Z}+\varepsilon
$$

où $\mathbf{Z}$ est un vecteur comportant des caractéristiques spécifiques du ménage et des facteurs de distribution susceptibles d'influencer la règle de partage. En particulier, ce vecteur contient la différence des salaires des deux conjoints $\left(w_{f}-w_{m}\right)$ et le revenu hors travail $y$. Parfois, le revenu exogène $y$ peut être individualisé, et les revenus hors travail individuels sont souvent utilisés comme des facteurs de distribution (cf. Clark, Couprie et Sofer, 2004) ${ }^{8}$.

Les valeurs prises par l'indice d'inégalité intrafamiliale peuvent être spécifiées comme suit :

$$
\mathrm{I}_{3}=\left\{\begin{array}{l}
0, \text { si } \Phi^{*} \leq \kappa_{1}, \\
1, \text { si } \kappa_{1}<\Phi^{*} \leq \kappa_{2}, \\
2, \text { si } \Phi^{*}>\kappa_{2},
\end{array}\right.
$$

où $k_{1}$ et $k_{2}$ sont des paramètres à estimer.

Rappelons à nouveau que la règle de partage $\Phi_{f}$ dépend du profit tiré de la production domestique $\Pi$ qui est endogène au modèle, car la production domestique dépend à son tour du temps passé au travail domestique et des taux de salaire. Donc, le système (7) est complété par deux équations décrivant le travail domestique des conjoints appelées $t_{f}$ et $t_{m}$ pour le travail domestique de la femme et de l'homme respectivement (les logarithmes népériens des heures de travail domestique mensuelles). Le système final (8) est la représentation économétrique du modèle théorique (5) :

$$
\left\{\begin{array}{l}
\mathrm{I}_{3}=0, \text { si } \Phi^{*} \leq \kappa_{1}, \\
\mathrm{I}_{3}=1, \text { si } \kappa_{1}<\Phi^{*} \leq \kappa_{2}, \\
\mathrm{I}_{3}=2, \text { si } \Phi^{*}>\kappa_{2}, \\
t_{f}=\boldsymbol{\alpha}_{f} \boldsymbol{X}_{f}+u_{1} \\
t_{m}=\boldsymbol{\alpha}_{m} \boldsymbol{X}_{m}+u_{2}
\end{array}\right.
$$

\footnotetext{
${ }^{8}$ Ici, dans les données RLMS, bien que l'individualisation des revenus hors travail soit, en principe, possible, la variable s'est révélée trop peu fiable pour être utilisable en pratique.
} 
où $\boldsymbol{\alpha}_{i}(\mathrm{i}=\mathrm{f}, \mathrm{m})$ sont les vecteurs de paramètres, $\mathbf{X}_{i}(\mathrm{i}=\mathrm{f}, \mathrm{m})$ sont les vecteurs des caractéristiques individuelles et du ménage. Les termes d'erreur $\varepsilon, u_{1}, u_{2}$ ont une distribution standard normale trivariée de moyenne nulle et une matrice des variances covariances $\boldsymbol{\Sigma}$ :

$$
\Sigma=\left[\begin{array}{ccc}
1 & \sigma_{\varepsilon u_{1}} & \sigma_{\varepsilon u_{2}} \\
\sigma_{\varepsilon u_{1}} & \sigma_{1}^{2} & \sigma_{u_{1} u_{2}} \\
\sigma_{\varepsilon u_{2}} & \sigma_{u_{1} u_{2}} & \sigma_{2}^{2}
\end{array}\right]
$$

$\operatorname{avec} \sigma_{\varepsilon u_{j}}=\operatorname{cov}\left(\varepsilon, u_{j}\right), \sigma_{u_{1} u_{2}}=\operatorname{cov}\left(u_{1}, u_{2}\right), j=1,2, \sigma_{1}^{2}=\operatorname{Var}\left(u_{1}\right)$ et $\sigma_{2}^{2}=\operatorname{Var}\left(u_{2}\right)$

Le modèle est estimé avec une méthode qui permet d'estimer simultanément trois équations : le probit ordonné pour l'indice de l'inégalité intrafamiliale, et deux équations avec des variables dépendantes continues pour les heures de travail domestique des deux conjoints.

La fonction de vraisemblance pour le système d'équation (8) est la suivante :

$$
\begin{aligned}
L=\prod_{i: I=0} & {\left[F\left(\left(\kappa_{1}-\gamma_{i}{ }^{\prime} \mathbf{Z}_{i}\right) \mid u_{1 i}, u_{2 i}\right) \times f\left(u_{1 i}, u_{2 i}\right)\right] \times } \\
& \times \prod_{i: I=1}\left[\left(F\left(\left(\kappa_{2}-\gamma_{i}{ }^{\prime} \mathbf{Z}_{i}\right) \mid u_{1}, u_{2}\right)-F\left(\left(\kappa_{1}-\gamma_{i}{ }^{\prime} \mathbf{Z}_{i}\right) \mid u_{1 i}, u_{2 i}\right)\right) \times f\left(u_{1 i}, u_{2 i}\right)\right] \times \\
& \times \prod_{i: I=2}\left[1-F\left(\left(\kappa_{2}-\gamma_{i}{ }^{\prime} \mathbf{Z}_{i}\right) \mid u_{1 i}, u_{2 i}\right) \times f\left(u_{1 i}, u_{2 i}\right)\right]
\end{aligned}
$$

où $i$ se réfère à l'observation $i, F\left(. u_{1 i}, u_{2 i}\right)$ est une fonction de distribution conditionnelle cumulée de $\varepsilon$ sur $u_{1 i}, u_{2 i} ; f\left(u_{1 i}, u_{2 i}\right)$ est une fonction de la distribution standard normale bivariée.

La variable $\varepsilon \mid u_{1}, u_{2}$ suit une distribution normale. En notant :

$$
\widetilde{\Sigma}=\left[\begin{array}{cc}
\sigma_{1}^{2} & \sigma_{u_{1} u_{2}} \\
\sigma_{u_{1} u_{2}} & \sigma_{2}^{2}
\end{array}\right]
$$

nous trouvons sa moyenne $\mu$ et sa variance $\sigma^{2}$ selon les formules suivantes (Green, 2000) :

$$
\begin{aligned}
& \mu=\left(\sigma_{\varepsilon u_{1}}, \sigma_{\varepsilon u_{2}}\right) \widetilde{\Sigma}^{-1}\left(u_{1}, u_{2}\right)^{\prime}=\left[\left(\rho_{1} u_{1} / \sigma_{1}+\rho_{2} u_{2} / \sigma_{2}\right)-\rho\left(\rho_{1} u_{2} / \sigma_{2}+\rho_{2} u_{1} / \sigma_{1}\right)\right] /\left(1-\rho^{2}\right) \\
& \sigma^{2}=1-\left(\sigma_{\varepsilon u_{1}}, \sigma_{\varepsilon u_{2}}\right) \widetilde{\Sigma}^{-1}\left(\sigma_{\varepsilon u_{1}}, \sigma_{\varepsilon u_{2}}\right)^{\prime}=1-\left\lfloor\rho_{1}^{2}+\rho_{2}^{2}-2 \rho \rho_{1} \rho_{2}\right\rfloor /\left(1-\rho^{2}\right),
\end{aligned}
$$

où $\rho_{1}, \rho_{2}, \rho$ sont des coefficients de corrélation entre $\varepsilon$ et $u_{1}, \varepsilon$ et $u_{2}, u_{1}$ et $u_{2}$ respectivement.

Donc, la fonction logarithmique de vraisemblance peut être définie dans les termes d'une distribution standard normale cumulée comme suit : 


$$
\ln L=\sum_{i: I=0} \ln F_{0}\left(z_{i}^{1}\right) \times f\left(u_{1 i}, u_{2 i}\right)+\sum_{i: I=1} \ln \left(F_{0}\left(z_{i}^{2}\right)-F_{0}\left(z_{i}^{1}\right)\right) \times f\left(u_{1 i}, u_{2 i}\right)+\sum_{i: I=0} \ln \left(1-F_{0}\left(z_{i}^{2}\right)\right) \times f\left(u_{1 i}, u_{2 i}\right)
$$

$\operatorname{avec} F_{0}$ la fonction de distribution standard normale cumulée et $z_{i}^{j}=\left(k_{j}-\gamma_{i}{ }^{\prime} \mathbf{Z}_{i}-\mu\right) / \sigma,(j=1,2)$.

Les effets marginaux des variables continues sur les probabilité d'une inégalité au profit de l'homme $\left(\partial P(0) / \partial X_{3}\right)$, de l'égalité $\left(\partial P(1) / \partial X_{3}\right)$ ou d'une inégalité au profit de la femme $\left(\partial P(2) / \partial X_{3}\right)$ sont calculées comme suit :

$$
\begin{aligned}
& \frac{\partial P(0)}{\partial X_{3}}=-f\left(k_{1}-\gamma \mathbf{Z}\right) \frac{\partial(\gamma \mathbf{Z})}{\partial X_{3}} \\
& \frac{\partial P(1)}{\partial X_{3}}=\left[f\left(k_{1}-\gamma \mathbf{Z}\right)-f\left(k_{2}-\gamma \mathbf{Z}\right)\right] \frac{\partial(\gamma \mathbf{Z})}{\partial X_{3}} \\
& \frac{\partial P(0)}{\partial X_{3}}=f\left(k_{2}-\gamma \mathbf{Z}\right) \frac{\partial(\gamma \mathbf{Z})}{\partial X_{3}}
\end{aligned}
$$

Le terme $\gamma \mathbf{Z}$ présent dans les arguments des fonctions de densité se calcule aux valeurs moyennes de l'échantillon. Le terme $\frac{\partial(\gamma \mathbf{Z})}{\partial X_{3}}$ est le coefficient correspondant obtenu par l'estimation pour les variables rentrant dans l'équation de la variable latente de façon linéaire. Pour les variables rentrant dans l'équation de manière non linéaire, ce terme se calcule de la façon suivante :

$$
\begin{aligned}
& \frac{\partial(\gamma \mathbf{Z})}{\partial \ln \left(w_{m}\right)}=\gamma_{2}-\gamma_{1} \\
& \frac{\partial(\gamma \mathbf{Z})}{\partial\left(w_{f} / w_{m}\right)}=\gamma_{1} \frac{w_{f}}{w_{m}} \\
& \frac{\partial(\gamma \mathbf{Z})}{\partial w_{f}}=\gamma_{1} \frac{1}{w_{f}} \quad \frac{\partial(\gamma \mathbf{Z})}{\partial w_{m}}=\left(\gamma_{2}-\gamma_{1}\right) \frac{1}{w_{m}}
\end{aligned}
$$

où $\gamma_{1}$ et $\gamma_{2}$ sont les coefficients correspondant aux variables $\ln \left(\frac{w_{f}}{w_{m}}\right)$ et $\ln \left(w_{m}\right)$ respectivement.

Pour les variables dummy $D_{3}$ les effets marginaux sont calculés comme les différences entre les probabilités correspondant à deux valeurs différentes de ces variables, les autres variables étant fixées à leur valeur moyenne dans l'échantillon :

$$
\begin{aligned}
& \frac{\partial P(0)}{\partial D_{3}}=\left[F\left(k_{1}-\boldsymbol{\gamma} \mathbf{Z}\right)\right]_{D_{3}=1}-\left[F\left(k_{1}-\gamma \mathbf{Z}\right)\right]_{D_{3}=0} \\
& \frac{\partial P(1)}{\partial D_{3}}=\left[F\left(k_{2}-\gamma \mathbf{Z}\right)-F\left(k_{1}-\gamma \mathbf{Z}\right)\right]_{D_{3}=1}-\left[F\left(k_{2}-\gamma \mathbf{Z}\right)-F\left(k_{1}-\gamma \mathbf{Z}\right)\right]_{D_{3}=0} \\
& \frac{\partial P(0)}{\partial D_{3}}=\left[F\left(k_{2}-\gamma \mathbf{Z}\right)\right]_{D_{3}=1}-\left[F\left(k_{2}-\gamma \mathbf{Z}\right)\right]_{D_{3}=0}
\end{aligned}
$$




\subsection{Les résultats}

Les résultats du probit ordonné apparaissent dans le tableau 10 ci-dessous :

Tableau 10. Estimation du différentiel des revenus (Indice $\mathrm{I}_{1}$ ) par un probit ordonné simple

\begin{tabular}{|l|c|}
\hline & Indice d'inégalité $\mathrm{I}_{1}{ }^{\mathrm{a}}$ \\
\cline { 2 - 2 } Ln de taux de salaire de l'homme & Coefficient \\
Ln du rapport des salaires : $\ln \left(w_{f} / w_{m}\right)$ & $0.073^{* *}$ \\
Age de l'homme & $0,158^{* * *}$ \\
Différence d'âge (age -age $_{\mathrm{m}}$ ) & $-0,002$ \\
Sex ratio & $-0,017^{* * *}$ \\
Revenu hors travail du ménage & $-0,132$ \\
Nombre d'années d'éducation de l'homme & -0.0002 \\
La femme est plus éduquée que l'homme (dummy) & -0.009 \\
& $0.098^{*}$ \\
Nombre d'enfants entre 0 et 7 ans & $-0,042$ \\
Nombre d'enfants entre 8 et 18 ans & 0,009 \\
Nombre de personnes âgées & 0,069 \\
Ln de la taille du logement (en mètres carrés) & -0.021 \\
Ménage travaille sur un lopin de terre & -0.15 \\
Moscou/Saint-Pétersbourg & -0.064 \\
Vague5 & $0.114^{*}$ \\
Vague6 & 0.069 \\
Vague8 & 0.019 \\
Paramètres auxiliaires & \\
$k_{1}$ & \\
$k_{2}$ & $-1.39 * * *$ \\
$k_{3}$ & $-0.636^{* *}$ \\
$k_{4}$ & $0,461^{*}$ \\
Nombre d'observations & $1,186^{* * *}$ \\
Log vraisemblance & 2174 \\
significatif à 10\%; $* *$ significatif à 5\%; *** significatif à 1\% & $-3154,606$ \\
Source : RLMS (vagues 5-8) & \\
\hline
\end{tabular}

On peut constater tout d'abord que l'estimation des seuils donne des résultats très significatifs, confortant ainsi la méthode utilisée. On constate également que la variable dont on pouvait bien attendre intuitivement qu'elle ait un rôle majeur sur le pouvoir de négociation, à savoir le rapport des salaires entre conjoints a un effet très significatif dans le sens attendu : plus le salaire de la femme est élevé relativement au salaire du mari, et plus elle déclare un revenu élevé relativement à celui déclaré par son conjoint. Les résultats principaux du modèle collectif trouvent donc là une confirmation claire. Cet effet est d'autant plus fort que les conjoints ont un taux de salaire élevé. Comme variable de distribution nous avons inclus le sex ratio qui est trouvé, comme bien souvent, non significatif (cf, par exemple, Clark Couprie et Sofer, 2004). Pour la construction de cette variable nous avons utilisé les données officielles russes (Goskomstat, 2001) du nombre de femmes et d'hommes par âge, par région 
et par période. Plus précisément pour chaque ménage le sex ratio est égal au nombre d'hommes pour le groupe d'âge de la femme divisé par le nombre de femmes pour le groupe d'âge de l'homme, le groupe d'âge étant défini par tranches de 4 années. Le marché du mariage semble donc bien suivre des règles un peu plus subtiles qu'une réaction au rapport brut de tous les hommes de la classe d'âge de la femme rapporté au nombre de femmes de la classe d'âge de l'homme, d'autant que, du moins en Russie comme en Europe, la variabilité de ce rapport est en général faible d'une région à l'autre. En revanche, l'écart d'âge entre les conjoints (âge de la femme moins âge de l'homme) a un effet négatif significatif : les femmes relativement plus jeunes que leur mari ont un pouvoir de négociation plus fort, ce qui peut bien s'interpréter comme un avantage comparatif sur le marché du mariage. Relevons également l'effet positif sur le pouvoir de négociation de la femme d'un différentiel en sa faveur dans le niveau d'éducation. Là encore, l'interprétation en termes de pouvoir de négociation est immédiate : toutes choses égales par ailleurs (en particulier les salaires), une femme plus éduquée est mieux capable de faire valoir son point de vue dans le ménage qu'une femme au niveau d'éducation relatif plus faible. Ces premiers résultats tendent donc bien à confirmer une approche du ménage en termes de pouvoir de négociation.

Examinons maintenant les résultats du modèle avec travail domestique endogène. Ils apparaissent dans le tableau 11 ci-dessous. Les trois variables dépendantes pour cette estimation sont les suivantes: le logarithme népérien de la valeur du travail domestique mensuel de l'homme et de sa conjointe, et la valeur de l'indice d'inégalité intrafamiliale (indice $\mathrm{I}_{3}$ ). Par rapport au modèle précédent, nous avons enlevé un certain nombre de variables qui n'apparaissent significatives dans aucune des équations, comme le sex ratio par exemple. Par ailleurs, le nombre d'observations dans le tableau 11 est moins élevé que le nombre d'observations dans le tableau précédent (1916 versus 2174). Ceci est dû à l'existence de valeurs manquantes ainsi que de valeurs nulles pour les heures du travail domestique de chaque époux.

Le test clé de la spécification du modèle probit ordonné est celui de la normalité des résidus. Nous avons appliqué une généralisation du test de Bera, Jarque, Lee (1984) proposé par Johnson (1995) pour les modèles de probit ordonné. La statistique du test est $\chi^{2}(2)=$ 0.719712346, ce qui donne la probabilité de test Prob $>\chi^{2}=0.7$. On ne peut pas donc rejeter l'hypothèse $\mathrm{H}_{0}$ de normalité des erreurs. La construction de la statistique est expliquée en annexe. 
Tableau 11 Estimation des offres du travail domestique de deux conjoints et de l'indice de l'inégalité intra-familiale $\mathrm{I}_{3}{ }^{\mathrm{a}}$ par la méthode du Maximum de Vraisemblance.

\begin{tabular}{|c|c|c|c|c|c|c|}
\hline & \multirow{2}{*}{$\begin{array}{c}\text { Temps de travail } \\
\text { domestique de la } \\
\text { femme } \\
\text { Coefficient }\end{array}$} & \multirow{2}{*}{$\begin{array}{c}\begin{array}{c}\text { Temps de travail } \\
\text { domestique de } \\
\text { l'homme }\end{array} \\
\text { Coefficient }\end{array}$} & \multirow{2}{*}{$\begin{array}{c}\text { Indice } \\
\text { d'inégalité } \\
I^{\mathrm{a}} \\
\text { Coefficient }\end{array}$} & \multicolumn{3}{|c|}{ Effets marginaux issus du probit ordonné } \\
\hline & & & & $\partial P(0) / \partial X_{3}$ & $\partial P(1) / \partial X_{3}$ & $\partial P(2) / \partial X_{3}$ \\
\hline Ln de taux de salaire de l'homme & 0,019 & 0,034 & $0,063^{*}$ & 0,0168 & $-0,0013$ & $-0,0155$ \\
\hline Ln de taux de salaire de la femme & 0,023 & $-0,004$ & & $-0,0282$ & 0,0022 & 0,0259 \\
\hline $\mathrm{Ln}\left(\mathrm{w}_{\mathrm{f}} / \mathrm{w}_{\mathrm{m}}\right)^{\mathrm{b}}$ & & & $0,156 * * *$ & $-0,0109$ & 0,0008 & 0,0100 \\
\hline Taux de salaire de l'homme & & & & 0,0013 & $-0,0001$ & $-0,0012$ \\
\hline Taux de salaire de la femme & & & & $-0,0037$ & 0,0003 & 0,0034 \\
\hline $\mathrm{w}_{\mathrm{f}} / \mathrm{w}_{\mathrm{m}}$ & & & & $-0,0173$ & 0,0014 & 0,0159 \\
\hline Age de l'homme & & $-0,009$ & & & & \\
\hline Age de l'homme au carré & & 0,004 & & & & \\
\hline Age de la femme & 0,020 & & & & & \\
\hline Age de la femme au carré & $-0,018$ & & & & & \\
\hline $\mathrm{Age}_{\mathrm{f}}-\mathrm{age}_{\mathrm{m}}^{\mathrm{c}}$ & & & $-0,016 * *$ & 0,0028 & $-0,0002$ & $-0,0026$ \\
\hline $\begin{array}{l}\text { Femme a une éducation technique ou } \\
\text { supérieure }\end{array}$ & 0,016 & & & & & \\
\hline $\begin{array}{l}\text { Homme a une éducation technique ou } \\
\text { supérieure }\end{array}$ & & $0,130^{*}$ & & & & \\
\hline Nombre d'années d'éducation de l'homme & & & $-0,018 *$ & 0,00005 & $-0,00000$ & $-0,0000$ \\
\hline Education $_{\mathrm{f}}>$ Education $_{\mathrm{m}}$ & & & 0,044 & $-0,0099$ & 0,0198 & $-0,0099$ \\
\hline Revenu hors travail du ménage & & & 0,000 & 0,0013 & $-0,0001$ & $-0,0012$ \\
\hline Nombre d'enfants entre 0 et 7 ans & $0,444 * * *$ & $0,666 * * *$ & $-0,008$ & $-0,0096$ & 0,0007 & 0,0088 \\
\hline Nombre d'enfants entre 8 et 18 ans & $0,199 * * *$ & $0,273 * * *$ & 0,053 & $-0,0094$ & 0,0007 & 0,0086 \\
\hline Nombre de personnes âgées & 0,008 & 0,110 & 0,052 & 0,0012 & $-0,0001$ & $-0,0011$ \\
\hline Ln de la taille du logement (en mètres carrés) & $-0,090^{*}$ & $-0,138$ & $-0,007$ & 0,0008 & $-0,0000$ & $-0,0007$ \\
\hline Possession d'une voiture & 0,021 & 0,029 & & & & \\
\hline Possession d'une machine à laver & $-0,056$ & 0,007 & & & & \\
\hline Ménage travaille sur un lopin de terre & $-0,015$ & 0,042 & $-0,005$ & 0,0010 & $-0,0020$ & 0,0010 \\
\hline Habitat rural & $0,162 * * *$ & 0,170 & & & & \\
\hline Caucase & $-0,068$ & 0,120 & & & & \\
\hline Volga & $-0,048$ & $-0,099$ & & & & \\
\hline Moscou/Saint-Pétersbourg & $-0,102 *$ & $-0,058$ & $-0,028$ & 0,0064 & $-0,01251$ & 0,0060 \\
\hline Nord-ouest & $-0,082$ & 0,202 & & & & \\
\hline Oural & $-0,227 * * *$ & $-0,104$ & & & & \\
\hline Sibérie Occidentale & $-0,137 * *$ & $-0,222$ & & & & \\
\hline Sibérie Orientale & $-0,192 * * *$ & $-0,029$ & & & & \\
\hline Vague5 & $0,119 * * *$ & $0,232 * * *$ & 0,089 & $-0,0198$ & 0,0397 & $-0,0198$ \\
\hline Vague6 & $-0,006$ & $-0,110$ & 0,038 & $-0,0086$ & 0,0171 & $-0,0085$ \\
\hline Vague8 & $-0,044$ & $-0,104$ & $-0,012$ & 0,0026 & $-0,0051$ & 0,0025 \\
\hline Constante & $4,489 * * *$ & $3,448 * * *$ & & & & \\
\hline Paramètres auxiliaires & & & & & & \\
\hline$k_{1}$ & & & $-1,275 * * *$ & & & \\
\hline$k_{2}$ & & & $1,304 * * *$ & & & \\
\hline $\begin{array}{l}\rho_{1} \text { (corrélation entre l'offre de travail } \\
\text { domestique de la femme et la règle de partage) }\end{array}$ & & -0.041 & & & & \\
\hline $\begin{array}{l}\rho_{2} \text { (corrélation entre l'offre de travail } \\
\text { domestique de l'homme et la règle de partage) }\end{array}$ & & -0.016 & & & & \\
\hline$\rho$ (corrélation entre l'offre de travail & & $0.198 * * *$ & & & & \\
\hline domestique de l'homme et celle de la femme) & & & & & & \\
\hline$\sigma_{1}$ & & $0,757 * * *$ & & & & \\
\hline$\sigma_{2}$ & & $1.465 * * *$ & & & & \\
\hline Nombre d'observations & & 2177 & & & & \\
\hline Log vraisemblance & & $-7749,477$ & & & & \\
\hline
\end{tabular}

* significatif à $10 \% ; * *$ significatif à $5 \% ; * * *$ significatif à $1 \%$

${ }^{\text {a }}$ La variable dépendante est égale à 0 si la réponse de la femme se situe au moins deux paliers en dessous de celle de son mari (la femme se sent plus pauvre que son mari); elle est égale à 1 si la différence est nulle ou égale à 1 en valeur absolue (les deux conjoints déclarent des revenus proches, c'est-à-dire égaux à un palier près); enfin, elle est égale à 2 si la réponse de la femme se situe au moins deux paliers au dessus de celle de son mari (la femme se sent plus riche que son mari).

Variables de référence : urbain, région Centre, vague 7 de l'observation

${ }^{\mathrm{b}} \ln w_{f}-\ln w_{m}$

${ }^{\mathrm{c}}$ L'âge de la femme moins l'âge de l'homme

Source : RLMS (vagues 5-8) 
Les variables exerçant une influence significative sur l'offre de travail domestique ne sont pas nombreuses. Les taux de salaire, tant masculin que féminin, ne semblent pas jouer de rôle déterminant sur l'activité domestique. Une variable qui paraît influencer (positivement) le temps de travail domestique de l'homme est son niveau d'éducation. On peut penser qu'un niveau d'éducation élevé favorise un partage des tâches plus égalitaire, et il s'agit d'un résultat souvent trouvé aussi dans d'autres pays. Les variables représentant le nombre de jeunes enfants (âgés de 0 à 7 ans) et d'enfants plus grands (âgés de 8 à 18 ans) sont significatives dans les deux équations. Les résultats sont conformes à ce qu'on attendait : un plus grand nombre d'enfants (surtout de jeunes enfants) augmente le nombre d'heures dédiées au travail domestique. Les caractéristiques non liées au marché du travail ne sont, elles, pas souvent significatives : en dehors de la taille du logement, mais qui exercerait plutôt un effet négatif sur le travail domestique de la femme (est-ce parce qu'un logement plus grand correspond à un nombre plus grand de personnes dans le ménage, et donc éventuellement à une aide ménagère de la part d'autres adultes ?), la possession de biens durables ou d'un lopin de terre n'exercent aucune influence sur la durée du travail domestique des conjoints. En revanche, le type de résidence et quelques régions d'habitation sont significatifs. Les hommes et les femmes vivant en milieu rural ont tendance à travailler plus à la maison que les habitants des villes. Par rapport à la région Centre, les conjoints (les hommes et les femmes) effectuent moins de travail domestique à Moscou et Saint-Pétersbourg, et les femmes travaillent moins dans l'Oural, en Sibérie Occidentale et Orientale.

Dans l'équation du probit ordonné, les résultats sont proches de ceux du tableau 10. Parmi les résultats similaires, notons évidemment tout d'abord l'effet des salaires relatifs. Ce résultat est confirmé par l'analyse des effets marginaux (cf. $2^{\text {ème }}$ partie du tableau 11 ci-dessus i.e. trois dernières colonnes). Les effets marginaux sont presque équivalents en valeur absolue pour la première et la dernière colonne de la $2^{\text {ème }}$ partie du tableau 11 correspondant à un écart de 2 paliers ou plus : les résultats sont donc symétriques et de signe opposé pour les situations où la femme se situe loin de son conjoint en termes de revenu. Le ratio entre les taux de salaire est donc un facteur déterminant dans l'explication du processus de négociation. Ceci est bien conforme aux prédictions du modèle collectif et justifie le choix de ce type de modèle pour l'analyse de la distribution des revenus à l'intérieur du ménage. En effet, si contrairement à l'hypothèse du modèle collectif, on choisissait plutôt le modèle unitaire, alors le revenu de chaque membre de ménage serait le même (revenu total) et les divergences des réponses à la question sur le revenu montreraient simplement des différences subjectives aléatoires entre les 
conjoints et donc ne seraient pas liées avec les salaires d'une façon significative. Ainsi notre test empirique permet d'accepter l'hypothèse de partage du revenu à la suite d'un processus de négociation.

Nous retrouvons également qu'une autre variable influençant de manière significative le partage du revenu total à l'intérieur du ménage est la différence d'âge entre les conjoints. Nous ne trouvons plus après correction, en revanche, d'effet significatif de la différence d'éducation entre les deux conjoints, même si un niveau d'éducation plus élevé de l'homme semble bien, toutes choses égales par ailleurs (et en particulier les salaires des conjoints), favoriser la femme. Notons ici que l'effet du nombre d'enfants sur la règle de partage ne peut être prédit théoriquement de façon évidente : d'une part, un nombre d'enfants élevé tend à affaiblir la position de la femme sur le marché du remariage. Mais, à l'inverse, et c'est plutôt la version que véhiculent la sociologie ou l'anthropologie, un nombre d'enfant plus élevé peut accroître le pouvoir de négociation de leur mère, par exemple via l'accroissement de la valeur de la production domestique qu'elle fournit (les substituts marchands à la garde et à l'éducation des enfants sont relativement chers). L'effet final est donc indéterminé, ce qui peut expliquer la non significativité des variables correspondant à la présence d'enfants

Notons que la corrélation entre l'offre de travail domestique de chaque conjoint et la règle de partage est très faible et non significative aussi bien dans le cas du travail domestique de l'homme que dans le cas de celui de la femme (cf. tableau 11, coefficients $\rho_{1}$ et $\rho_{2}$ ). Nous avons vu que, dans le modèle théorique, ces corrélations peuvent apparaître via le profit de la production domestique si on fait l'hypothèse que seules les variables monétaires ou les facteurs de distribution influencent la règle de partage. Il s'agirait dans ce cas d'une influence indirecte du travail domestique sur la règle de partage, qui se manifeste à travers la répartition du temps de travail de la femme entre travail marchand et domestique et s'exerce par l'intermédiaire du profit. Nos résultats indiqueraient alors qu'une durée de travail domestique particulièrement élevée de la femme n'augmente que peu le profit tiré de la production domestique en comparaison avec ce qu'apporterait un supplément de travail marchand. Nos résultats devraient inciter en toute hypothèse à explorer plus avant cette possibilité (cf les travaux dans ce sens de Rapoport, Sofer, Solaz (2003) ou Bourguignon et Chiuri (2004). 


\section{Conclusion.}

Dans cette étude, nous avons proposé un test du modèle collectif ou, plus généralement, d'un modèle de négociation, pour l'analyse de l'inégalité intrafamiliale en utilisant les réponses à une question subjective sur le niveau de revenu. Nous utilisons ici le cadre théorique du modèle collectif avec production domestique. Les résultats de notre analyse montrent tout d'abord que plus de la moitié des ménages divergent sur la réponse donnée. Une minorité non négligeable diverge même très sensiblement (au moins deux paliers d'écart sur une échelle de neuf). Ils montrent ensuite que les divergences dans les réponses, loin de refléter simplement des différences subjectives aléatoires entre les conjoints, correspondent bien de manière significative aux prédictions du modèle collectif : le rapport des salaires semble bien être un facteur déterminant de la règle de partage, donc de l'inégalité intrafamiliale. Ceci est bien conforme aux prédictions du modèle collectif et justifie le choix de ce type de modèle pour l'analyse de la distribution des revenus à l'intérieur du ménage. Par ailleurs, la prise en compte de facteurs de distribution tels que le sex ratio ou la différence d'âge des conjoints révèle bien un effet significatif de cette dernière variable (en faveur des femmes plus jeunes), mais en revanche aucun effet significatif du sex ratio. Enfin, la mise en évidence d'un effet positif du nombre d'enfant sur le revenu alloué à leur mère, ou encore la corrélation, faible mais significative, trouvée entre le temps de travail domestique de la femme et sa part de revenu plaident en faveur d'une analyse plus fine des effets potentiels des variables liées à des caractéristiques non marchandes sur la règle de partage. Notons pour conclure qu'un prolongement naturel de ce travail pourrait être de réaliser une identification de la règle de partage elle-même. 


\section{Bibliographie}

Apps P. et Rees R. (1997), "Collective Labor Supply and Household Production", Journal of Political Economy, 105, pp. 178-190.

Apps P. et Rees R. (1999), " Individual versus Joint Taxation in Models with Household Production », The Journal of Political Economy, 107, pp. 393-403.

Aronsson T., Daunfeldt S.-O. et Wickström M. (2001), « Estimating Intra-Household Allocation in a collective Model with Household Production », Journal of Population Economics, 14, pp. 569-584.

Bera A.K., Jarque C.M., Lee L.-F. (1984), "Testing the Normality Assumption in Limited Dependent Variable Models", International Economic Review, 25, 563-578.

Blundell R., Chiappori P.A., Magnac T. et Meghir C. (1998) "Collective labor supply: heterogeneity and non participation", Mimeo, UCL.

Bonke et Browning M. (2003) "The distribution of well-being and income within the household", W.P. Centre for Applied Microeconometrics, Institute of Economics, University of Copenhagen.

Bourguignon F., Chiappori P.A. (1992), «Collective models of household behavior: An introduction », European Economic Review, 36, p.355-364.

Bourguignon F. et Chiuri M.C. (2005), "Labor Market Time and Home Production: a new test for collective models of intra-household allocation", CSEF Working Paper 131.

Browning M., Chiappori P.A. (1998), « Efficient intra-household allocations : a general characterization and empirical tests », Econometrica, 66, p.1241-1278.

Browning M., Chiappori P-A. et Lewbel A. (2004). "Estimating Consumption Economies of Scale, Adult Equivalence Scales, and Household Bargaining Power", mimeo.

Chiappori P.A. (1988), « Rational household labor supply », Econometrica, 56, p.63-89.

Chiappori P.A. (1992), " Collective Labor Supply and Welfare», Journal of Political Economy, 100, p.437-467.

Chiappori P-A. (1997). "Introducing Household Production in Collective Models of Labor Supply", Journal of Political Economy, 105, pp. 191-209.

Chiappori P-A. (1998). "Rational Household Labor Supply", Econometrica, 56, pp. 63-89.

Chiappori P-A., Fortin, B. et Lacroix G. (2002). "Marriage Market, Divorce Legislation and Household Labor Supply", Journal of Political Economy, 110, pp.37-72. 
Clark A., Couprie H. et Sofer C. (2004). "La modélisation collective de l'offre de travail. Mise en perspective et application aux données britanniques", Revue Economique, 55, $\mathrm{n}^{\circ} 4$, pp.767-789.

Clark A. et Oswald A. (1994). "Unhappiness and unemployment" Economic Journal, vol. 104, pp. 648-659.

Couprie H. (2003) "Time allocation within the Family: welfare implications of life in a couple", Document de Travail GREQAM, n02A23.

Donni O. (2003) "Collective household labor supply: Non-participation and income taxation", The Journal of Public Economics, 87, pp. 1179-1198.

Earle, J.S. et Sabirianova K. (2002) "How Late to Pay? Understanding Wage Arrears in Russia", Journal of Labor Economics, 20(3), pp. 661-707.

Ferrer-i-Carbonell A. (2002). "Subjective Questions to Measure Welfare and Well-being", Tinbergen Institute Discussion Paper, 2002-020/3.

Ferrer-i-Carbonell A. et Frijters P. (2004). "How important is methodology for the estimates of the determinants of happiness?", Economic Journal, July, pp. 641-659.

Fong Y. et Zhang J. (2001) "The Identification of Unobservable and Independent Spousal Leisure", Journal of Political Economy, 109(1), pp. 191-202.

Fortin B. et Lacroix G. (1997) "A Test of the Unitary and Collective Models of Household Labour Supply." Economic Journal, vol. 107, issue 443, pages 933-55.

Greene W. H. (2000). Econometric Analysis, Fourth Edn., Englewood Cliffs, Prentice-Hall.

Heeringa, S.G. (1997) "Russia Longitudinal Monitoring Survey. Sample Attrition, Replenishment, and Weighting in Rounds V-VII", Director Survey Design and Analysis Unit, Institute for Social Research, University of Michgan, Ann Arbor, MI 48106-1248.

Johnson P.A. (1996), "A Test of the Normality Assumption in the Ordered Probit Model", METRON, LIV: 213-21.

Lundberg S. et Pollak R. (1996), "Bargaining and Distribution in Marriage", Journal of Economic Perspectives, Vol. 10, n²4, pp. 139-158.

Kalugina, E. et Najman, B. (2003) "Travail et pauvreté en Russie: évaluations objectives et perceptions subjectives", Economie et Statistique, n 367, p. 83-100.

Manser M., Brown M. (1980), « Marriage and Household Decision Theory-A Bargaining Analysis », International Economic Review, 21, 21-34.

Mezentseva E.B. (2000) Feminizatsiya bednosti v Rossii. Sbornik dokladov, podgotovlennix dlia Vsemirnogo Banka./La féminisation de la pauvreté en Russie. Rapports préparés pour la Banque Mondiale, - M.: "Ves' Mir". 
Moreau N., Donni O. (2002), « Une estimation d'un modèle collectif d'offre de travail avec taxation», Annales d'Economie et Statistiques, 65, 55-83.

McElroy M., Horney M. (1981), « Nash-Bargained Decisions : Toward a Generalization of the Theory of Demand », International Economic Review, 22, 333-349.

Najman B. et Pailhé A. (2001), "Mobilité externe sur le marché du travail russe, 1994-1998", Revue Economique, vol. 52, n 4 , pp. 861-884

Ng Y-K. (1996) "Happiness surveys: Some comparability issues and an exploratory survey based on just perceivable increments", Social Indicators Research, 38(1), pp.1-27.

Rapoport B., Sofer C. et Solaz A. (2003). "Household Production in a Collective Model: Some New Results", TEAM Working Paper, Université Paris 1-Panthéon-Sorbonne.

Rapoport B., Sofer C. et Solaz A. (2006). "La production domestique dans les modèles collectifs", Actualité Economique, à paraître.

Ravallion M. et Lokshin M. (2000). "Who Wants to Redistribute? The Tunnel Effect in 1990s Russia", Journal of Public Economics, 76, pp. 87-104.

Senik C. (2002). "Que nous apprennent les données subjectives? Une application au lien entre revenu et bien-être", Working paper $\mathrm{N}^{\circ}$ 2002-20 DELTA.

Senik C. (2004) "When Information Dominates Comparison. Learning from Russian Subjective Panel Data", Journal of Public Economics, vol. 88, issues 9-10, pp. 2099-2133.

Senik, C. (2005). "What Can we Learn from Subjective Data? The Case of Income and WellBeing", Journal of Economic Surveys, 19 (1), pp. 43-63.

Van Praag B.M.S. (1991). "Ordinal and cardinal utility: an integration of the two dimensions of the welfare concept", Journal of Econometrics, vol. 50, pp.69-89.

Winkelmann L. et Winkelmann R. (1998). "Why are the Unemployed So Unhappy? Evidence from Panel Data", Economica, 65, pp. 1-15.

Winkelmann R. (2004). Subjective Well-being and the Family: Results from an Ordered Probit Model with Multiple Random Effects", Document du travail, l'Université de Zurich.

Zohoori, N., P. Kozyreva, M. Kosolapov, M. Swafford, B. Popkin, E. Glinskaya, M. Lokshin, and D. Mancini (1998) "Monitoring the Economic Transition in the Russian Federation and its Implications for the Demographic Crisis--the Russian Longitudinal Monitoring Survey." World Development 26(11), pp. 1977-1993. 


\section{ANNEXE}

Test de normalité des résidus de l'estimation du probit ordonné

Soit :

$s_{i 1}=\left\{\begin{array}{ll}1, & \text { si } \quad \Phi^{*}<k_{1} \\ 0, & \text { sinon }\end{array} \quad s_{i 2}=\left\{\begin{array}{ll}1, & \text { si } k_{1}<\Phi^{*}<k_{2} \\ 0, & \text { sinon }\end{array}, \quad s_{i 3}= \begin{cases}1, & \text { si } \quad \Phi^{*}>k_{2} \\ 0, & \text { sinon }\end{cases}\right.\right.$

$h_{i 1}=k_{1}-\gamma Z_{i} \quad h_{i 2}=k_{2}-\gamma Z_{i} \quad$ où $i$ désigne un ménage $i$.

$$
\begin{gathered}
\Sigma_{i}=\left(\begin{array}{l}
{\left[\frac{s_{i 1}}{F\left(h_{i 1}\right)}-\frac{s_{i 2}}{F\left(h_{i 2}\right)-F\left(h_{i 1}\right)}\right] f\left(h_{i 1}\right)} \\
{\left[\frac{s_{i 1}}{F\left(h_{i 1}\right)-F\left(h_{i 2}\right)}-\frac{s_{i 2}}{1-F\left(h_{i 2}\right)}\right] f\left(h_{i 2}\right)} \\
\sum_{j=1}^{3} s_{i j} \frac{f\left(h_{i j-1}\right)-f\left(h_{i j}\right)}{F\left(h_{i j}\right)-F\left(h_{i j-1}\right)} Z_{i} \\
\xi_{i}
\end{array}\right) \\
\xi_{i}=\left(\begin{array}{l}
\sum_{j=1}^{3} s_{i j} \frac{\left(1-h_{i j-1}^{2}\right) f\left(h_{i j-1}\right)-\left(1-h_{i j}^{2}\right) f\left(h_{i}\right)}{F\left(h_{i j}\right)-F\left(h_{i j-1}\right)} \\
\sum_{j=1}^{3} s_{i j} \frac{\left(3+h_{i j-1}^{2}\right) h_{i j-1} f\left(h_{i j-1}\right)-\left(3+h_{i j}^{2}\right) h_{i} f\left(h_{i}\right)}{F\left(h_{i j}\right)-F\left(h_{i j-1}\right)}
\end{array}\right)
\end{gathered}
$$

Alors la statistique LM (Lagrange multiplier) de l'hypothèse de normalité de $\varepsilon$ est calculée comme

$$
\nu=\left(\sum_{i=1}^{n} \xi_{i}\right)^{\prime} J^{\prime}\left(\sum_{i=1}^{n} \Sigma_{i} \Sigma_{i}^{\prime}\right)^{-1} J\left(\sum_{i=1}^{n} \xi_{i}\right)
$$

où $J$ est une matrice construite à partir des deux dernières colonnes d'une matrice identité de dimension $k+4$ avec $\mathrm{n}$ égal au nombre d'observations, $k$ égal au nombre de variables exogènes et $\Sigma_{i}$ estimée aux valeurs obtenues par oprobit MLE. $v$ est asymptotiquement distribuée comme une variable aléatoire de $\chi^{2}$ avec deux degrés de liberté. 
